\title{
Canonical Representations Generated by Translationally Quasi-invariant Measures
}

By

Hiroaki SHIMOMURA*

\section{§1. Introduction}

Let $G$ be a transformation group acting on some measurable space $(X, \mathfrak{B})$, and $\mu$ be a $G$-quasi-invariant probability measure. That is, $\mu_{g}$ defined by $\mu_{g}:=$ $\mu \circ g^{-1}$ is equivalent to $\mu\left(\mu_{g} \cong \mu\right)$ for all $g \in G$. Then a natural representation of $G$ in $\mathrm{L}_{\mu}^{2}$ arises such that,

$$
V_{\mu, \theta}(g): f(x) \in \mathrm{L}_{\mu}^{2} \longmapsto \sqrt{\frac{d \mu_{g}}{d \mu}} \theta(x, g) f\left(g^{-1} x\right) \in \mathrm{L}_{\mu}^{2},
$$

where $\theta(x, g)$ is a 1-cocycle.

We call it a canonical representation generated by $\mu$.

In this paper, $X$ is always a locally convex Hausdorff linear space over $\boldsymbol{R}$ and $\mathfrak{B}$ is the cylindrical $\sigma$-algebra on $X$. And $G$ consists of parallel displacement on $X, x \longmapsto x+\varphi$, where $\varphi$ runs through a linear subspace $\Phi$ of $X$. Up to the present time the representation of this type is considered together with the representation such type as

$$
\left.U_{\mu}(x): f(x) \in \mathrm{L}_{\mu}^{2} \longmapsto \exp \left(i<x, x^{*}\right\rangle\right) f(x) \in \mathrm{L}_{\mu}^{2},
$$

where $x^{*} \in X^{*}$ (topological dual space of $X$ ) in view of the field theory in quantum mechanics. However we shall treat here $V_{\mu, \theta}$ alone and discuss their various properties. The first important problem is a decomposition of these non irreducible representations. We shall carry out it using a direct integral of Hilbert spaces. This is one of main results of our subject and discussed in Section 2. If $\Phi$-quasi-invariant measure is also $\Psi$-quasi-invariant, then it becomes an interesting problem to discuss relations of the two representations of

Communicated by H. Araki, October 13, 1995. Revised April 1, 1996.

1991 Mathematics Subject Classification(s) : 60B11

*Department of Mathematics, Fukui University, Fukui 910, Japan. 
$\Phi$ and $\Psi$. These considerations not only clarify the theoretical structure, but also offer a technical tool assuring that some kinds of reasoning will be carried out smoothly. Those will be done in Section 3. Section 4 is a study of a $\Phi$-ergodic measure $\mu$, a spectral measure $\sigma$ and the multiplicity of the representation $V_{\mu, \theta}$. It will be shown that the ergodicity of $\mu$ derives that the spectral measure $\sigma$ is also $X^{*}$-ergodic and the representation $V_{\mu, \theta}$ has uniform multiplicity, and that the multiplicity 1 together with the $X^{*}$-ergodicity of $\sigma$ implies that $\mu$ is $\Phi$-ergodic. However we don't have a definite relation with these three relations, though it seems that there exists some interesting connection between multiplicity and ergodicity. Next we shall consider countable direct product of such representations $V_{\mu_{n}, \theta_{n}}(n=1,2, \cdots)$ in Section 5 and obtain a result like the theorem of Kakutani type. This is the second one of our main results. The third one discussed in Section 6 is a decision of maximal spectral type of Gaussian measures $\gamma$ on locally convex spaces. Moreover it will be turned out that under assumptions that $\theta$ is a trivial 1-cocycle or some special one, $V_{\gamma, \theta}$ is equivalent to $V_{\gamma^{\prime}, \theta^{\prime}}$, if and only if $\gamma$ is equivalent to some translation of $\gamma^{\prime}$.

\section{§2. Irreducible Decomposition of Canonical Representations by Direct Integrals}

2.1 Basic notation. Let $X$ be a locally convex Hausdorff linear space over $\mathbb{R}$ and $\mathfrak{B}$ be the cylindrical $\sigma$-algebra on $X$. That is, $\mathfrak{B}$ is the minimal $\sigma$-field with which all the continuous linear functionals $x^{*} \in X^{*}$ are measurable. Now we shall take $G$ as a translation group defined by some linear subspace $\Phi$ of $X$. So the probability measure which we are confronted with is $\Phi$-quasiinvariant one which is defined by a relation,

$$
\mu_{\varphi} \cong \mu
$$

for all $\varphi \in \Phi$, where $\mu_{\varphi}(\cdot)=\mu(\cdot-\varphi)$. In what follows we always assume that $\mathrm{L}_{\mu}^{2}$ is separable. Let $V_{\mu, \theta}$ be the canonical representation of $\Phi$ defined by

$$
V_{\mu, \theta}(\varphi): h(x) \in \mathrm{L}_{\mu}^{2} \longmapsto \sqrt{\frac{d \mu_{\varphi}}{d \mu}}(x) \theta(x, \varphi) h(x-\varphi) \in \mathrm{L}_{\mu}^{2},
$$

where $\theta$ is a 1-cocycle. That is, $\theta(x, \varphi)$ is a complex valued measurable function of $x$ for each fixed $\varphi \in \Phi$ whose absolute value is always 1 and

$$
\theta\left(x, \varphi_{2}\right) \theta\left(x-\varphi_{2}, \varphi_{1}\right)=\theta\left(x, \varphi_{1}+\varphi_{2}\right)
$$

for $\mu$-a.e. $x$, and

$$
\theta(x, 0)=1 \text {. }
$$

Now we shall demand that a one parameter group of operators $V_{\mu, \theta}(t \varphi)$ for each fixed $\varphi \in \Phi$ is continuous. Since 


$$
\left\|\sqrt{\frac{d \mu_{t \varphi}}{d \mu}}(x)-1\right\|_{2} \longrightarrow 0(t \rightarrow 0)
$$

(For example, see [9].), for the above requirement it is necessary and sufficient that

$$
\theta(x, t \varphi) \longrightarrow 0 \text { in } \mu \text {, as } t \rightarrow 0
$$

for each fixed $\varphi \in \Phi$. From now on we shall impose the above condition (2.4) upon $\theta$. We remark that (2.4) is immediately extended to an $n$-variable form,

$$
\begin{array}{r}
\theta\left(x, t_{1} \varphi_{1}+\cdots+t_{n} \varphi_{n}\right) \longrightarrow \theta\left(x, t_{1}^{\circ} \varphi_{1}+\cdots+t_{n}^{\circ} \varphi_{n}\right) \text { in } \mu, \\
\text { as }\left(t_{1}, \cdots, t_{n}\right) \longrightarrow\left(t_{1}^{\circ}, \cdots, t_{n}^{\circ}\right)
\end{array}
$$

for each fixed $\varphi_{1}, \cdots, \varphi_{n} \in \Phi$. Now $\left\langle V_{\mu, \theta}(\varphi) h, h\right\rangle_{2}\left(\langle\cdot, \cdot\rangle_{2}\right.$ is the inner product of $\mathrm{L}_{\mu}^{2}$.) is positive definite and continuous on any finite dimensional subspace of $\Phi$. Therefore the infinite dimensional Bochner's theorem assures an existence of $\sigma_{\mu, \theta, h} \equiv \sigma_{h}$ called an $h$-adjoint measure on a measurable space $\left(\Phi^{a}, \mho_{\Phi}\right)$ such that

$$
\widehat{\sigma_{h}}(\varphi):=\int_{\Phi^{a}} \exp \left(i\left\langle\varphi, \varphi^{a}\right\rangle\right) \sigma_{h}\left(d \varphi^{a}\right)=\left\langle V_{\mu, \theta}(\varphi) h, h\right\rangle_{2}
$$

where $\Phi^{a}$ is the algebraic dual space of $\Phi, \bigodot_{\Phi}$ is the minimal $\sigma$-algebra on $\Phi^{a}$ with which all the linear functionals, $\varphi^{a} \in \Phi^{a} \longmapsto\left\langle\varphi, \varphi^{a}\right\rangle \in \boldsymbol{R}$ are measurable.

Theorem 2.1. Let $d$ be the Hellinger distance defined on probability measures on $\left(\Phi^{a}, \mathfrak{\mho}_{\Phi}\right)$. Then we have

$$
d\left(\sigma_{h}, \sigma_{g}\right) \leq\|h-g\|_{2}
$$

for all $h, g \in \mathrm{L}_{\mu}^{2}$.

(The Hellinger distance is defined as follows;

$$
d^{2}\left(\sigma_{h}, \sigma_{g}\right):=\int_{\Phi^{a}}\left|\sqrt{\frac{d \sigma_{h}}{d p}}\left(\varphi^{a}\right)-\sqrt{\frac{d \sigma_{g}}{d p}}\left(\varphi^{a}\right)\right|^{2} p\left(d \varphi^{a}\right)
$$

where $p$ is an arbitrary $\sigma$-finite measure, as far as $\sigma_{h}$ and $\sigma_{g}$ is absolutely continuous with $p$.

Proof. We shall devide the proof into four steps.

(I) Put $L_{n}: \varphi^{a} \in \Phi^{a} \longmapsto\left(\left\langle\varphi_{1}, \varphi^{a}\right\rangle, \cdots,\left\langle\varphi_{n}, \varphi^{a}\right\rangle\right) \in \boldsymbol{R}^{n}$, where $\varphi_{1}, \cdots, \varphi_{n}$ is a linearly independent set of $\Phi$. We shall observe the explicit form of the image measure $L_{n} \sigma_{\mu, \theta, h}$. So let us decompose $X$ into a direct product using a dual system $\left\{\varphi_{j}^{*}\right\}\left(\varphi_{j}^{*} \in X^{*}\right.$ s.t., $\left.\left\langle\varphi_{k}, \varphi_{j}^{*}\right\rangle=\delta_{k, j}\right)$ as follows ;

$$
x \in X \longleftrightarrow\left(x_{1}, \cdots, x_{n}, \xi\right) \in \boldsymbol{R}^{n} \times\left(\Phi_{n}^{*}\right)^{\perp},
$$


where $x_{j}=\left\langle x, \varphi_{j}^{*}\right\rangle$ and $\xi=x-\sum_{j=1}^{n}\left\langle x, \varphi_{j}^{*}\right\rangle \varphi_{j}$ which will be denoted by $q_{n}(x)$. Since the natural measurable space $\left(\boldsymbol{R}^{n}, \mathfrak{B}\left(\boldsymbol{R}^{n}\right)\right)$ is standard, so any $\mu$ is decomposed into the regular conditional probabilities $\mu^{\xi}(\cdot)$ given $q_{n}=\xi$. Namely there exists a family of probability measures $\left\{\mu^{\xi}\right\}_{\xi \in\left(\Phi_{n}^{*}\right)^{\perp}}$ on $\left(\boldsymbol{R}^{n}, \mathfrak{B}\left(\boldsymbol{R}^{n}\right)\right)$ which satisfies,

$\mu^{\xi}(A)$ is a measurable function of $\xi \in\left(\Phi_{n}^{*}\right)^{\perp}$ for each fixed $A \in \mathfrak{B}\left(\mathbb{R}^{n}\right)$, (The measurable structure on $\left(\Phi_{n}^{*}\right)^{\perp}$ is an induced one from $(X, \mathfrak{B})$.) and

$$
\mu(A \times B)=\int_{B} \mu^{\xi}(A) q_{n} \mu(d \xi)
$$

for all $A \in \mathfrak{B}\left(\boldsymbol{R}^{n}\right)$ and all measurable sets $B \subseteq\left(\Phi_{n}^{*}\right)^{\perp}$.

As $\mu$ is $\Phi$-quasi-invariant, so $\mu^{\xi}$ is equivalent to the Lebesgue measure $\lambda_{n}$ on $\boldsymbol{R}^{n}$ for $q_{n} \mu$-a.e. $\xi$. Thus we have

$$
\mu \cong \lambda_{n} \times q_{n} \mu .
$$

Here we shall take a unitary map,

$$
T_{n}: h(x) \in \mathrm{L}_{\mu}^{2} \longmapsto \sqrt{\frac{d \mu}{d\left(\lambda_{n} \times q_{n} \mu\right)}}\left(x_{1}, \cdots, x_{n}, \xi\right) h\left(x_{1}, \cdots, x_{n}, \xi\right) \in \mathrm{L}_{\lambda_{n} \times q_{n} \mu}^{2}
$$

Then $V_{\mu, \theta}\left(t_{1} \varphi_{1}+\cdots+t_{n} \varphi_{n}\right)$ is converted as follows.

$$
\begin{aligned}
& T_{n} V_{\mu, \theta}\left(t_{1} \varphi_{1}+\cdots+t_{n} \varphi_{n}\right) T_{n}^{-1}: h\left(x_{1}, \cdots, x_{n}, \xi\right) \longmapsto \\
& \quad \theta\left(x_{1} \varphi_{1}+\cdots+x_{n} \varphi_{n}+\xi, t_{1} \varphi_{1}+\cdots+t_{n} \varphi_{n}\right) h\left(x_{1}-t_{1}, \cdots, x_{n}-t_{n}, \xi\right) .
\end{aligned}
$$

As $\theta$ is continuous in probability, so using the Slutsky's theorem (For example, see [4].) we deduce that there exists a jointly measurable function $\theta^{*}$ of $\left(\left(x_{1}, \cdots\right.\right.$, $\left.\left.x_{n}\right), \xi,\left(t_{1}, \cdots, t_{n}\right)\right)$ such that for each fixed $\left(t_{1}, \cdots, t_{n}\right) \in \boldsymbol{R}^{n}$,

$$
\theta^{*}\left(x_{1}, \cdots, x_{n}, \xi, t_{1}, \cdots, t_{n}\right)=\theta\left(x_{1} \varphi_{1}+\cdots+x_{n} \varphi_{n}+\xi, t_{1} \varphi_{1}+\cdots+t_{n} \varphi_{n}\right)
$$

for $\lambda_{n} \times q_{n} \mu$-a.e. $\left(x_{1}, \cdots, x_{n}, \xi\right)$.

(II) For a while we shall fix $\xi$ and shall write $x$ and $t$ instead of $\left(x_{1}, \cdots, x_{n}\right)$ and $\left(t_{1}, \cdots, t_{n}\right)$, respectively. Further we shall write $\theta_{\xi}^{*}(x, t)$ instead of $\theta^{*}(x, \xi, t)$. Then it follows from (2.2) that

$$
\theta_{\xi}^{*}(x, t)=\theta_{\xi}^{*}(x-t, s)^{-1} \theta_{\xi}^{*}(x, s+t)
$$

for $\lambda_{n} \times \lambda_{n} \times \lambda_{n}$-a.e. $(x, s, t)$. Here we shall change the variable $s$ to $\sigma=t+s-x$. Then (2.11) becomes

$$
\theta_{\xi}^{*}(x, t)=\theta_{\xi}^{*}(x-t, x+\sigma-t)^{-1} \theta_{\xi}^{*}(x, x+\sigma)
$$

for $\lambda_{n} \times \lambda_{n} \times \lambda_{n}$-a.e. $(x, t, \sigma)$, and therefore there exists a $\sigma_{0} \in \boldsymbol{R}^{n}$ such that

$$
\theta_{\xi}^{*}(x, t)=\theta_{\xi}^{*}\left(x-t, x-t+\sigma_{0}\right)^{-1} \theta_{\xi}^{*}\left(x, x+\sigma_{0}\right)
$$

for $\lambda_{n} \times \lambda_{n}$-a.e. $(x, t)$. We put 


$$
q_{n}(x, \xi):=\theta_{\xi}^{*}\left(x, x+\sigma_{0}\right)^{-1}
$$

Then it follows from (2.13) that

$$
\theta_{\xi}^{*}(x, \xi, t)=q_{n}(x-t, \xi) q_{n}(x, \xi)^{-1}
$$

for $\lambda_{n} \times q_{n} \mu \times \lambda_{n}$-a.e. $(x, \xi, t)$.

(III) We shall calculate the characteristic function of $L_{n} \sigma_{h}:=L_{n} \sigma_{\mu, \theta, h}$.

$$
\begin{aligned}
& \widehat{L_{n} \sigma_{h}}\left(t_{1}, \cdots, t_{n}\right) \\
& =\int_{\boldsymbol{R}^{n}} \exp \left(i \sum_{j=1}^{n} t_{j} y_{j}\right) L_{n} \sigma_{h}(d y) \\
& =\int_{\Phi^{a}} \exp \left(i \sum_{j=1}^{n} t_{j}\left\langle\varphi_{j}, \varphi^{a}\right\rangle\right) \sigma_{h}\left(d \varphi^{a}\right) \\
& =\left\langle V_{\mu, \theta}\left(\sum_{j=1}^{n} t_{j} \varphi_{j}\right) h, h\right\rangle_{2}=\left\langle T_{n} V_{\mu, \theta}\left(\sum_{j=1}^{n} t_{j} \varphi_{j}\right) T_{n}^{-1} T_{n} h, T_{n} h\right\rangle_{2} \\
& =\int_{\boldsymbol{R}^{n}} \int_{\left(\Phi_{n}^{*}\right)^{+}} \theta(x, \xi, t) T_{n} h(x-t, \xi) \overline{T_{n} h}(x, \xi) q_{n} \mu(d \xi) d x \\
& =\int_{\boldsymbol{R}^{n}} \int_{\left(\Phi_{n}^{*}\right)^{\perp}} \exp \left(i \sum_{j=1}^{n} t_{j} y_{j}\right)\left|\mathcal{F}\left(q_{n}(\cdot, \xi) T_{n} h(\cdot, \xi)\right)\right|^{2}\left(y_{1}, \cdots, y_{n}\right) q_{n} \mu(d \xi) d y,
\end{aligned}
$$

where $\mathscr{F}$ is the usual Fourier transform,

$$
(\mathscr{F} f)\left(y_{1}, \cdots, y_{n}\right)=(2 \pi)^{-n / 2} \int_{R^{n}} \exp \left(i \sum_{j=1}^{n} y_{j} x_{j}\right) f\left(x_{1}, \cdots, x_{n}\right) d x
$$

Note that the last equality holds for almost all $t=\left(t_{1}, \cdots, t_{n}\right)$. However each term appeared in this series of equalities are all continuous function of $t$, so it holds for every point. It follows that

$$
\frac{d L_{n} \sigma_{h}}{d y}=\int_{\left(\Phi_{n}^{*}\right)^{\perp}}\left|\mathcal{F}\left(q_{n}(\cdot, \xi) T_{n} h(\cdot, \xi)\right)\right|^{2}(y) q_{n} \mu(d \xi) .
$$

(IV) Put $p=2^{-1}\left(\sigma_{h}+\sigma_{g}\right)$. Then there exists a linearly independent at most countable set $\left\{\varphi_{1}, \cdots, \varphi_{n}, \cdots\right\} \subset \Phi$ such that both $\frac{d \sigma_{h}}{d p}\left(\varphi^{a}\right)$ and $\frac{d \sigma_{g}}{d p}\left(\varphi^{a}\right)$ are measurable with respect to $\mathbb{S}$, where $\mathbb{S}$ is the minimal $\sigma$-field with which all the functions $\left\langle\varphi_{n}, \varphi^{a}\right\rangle$ are measurable. If the suffix $n$ runs through $\{1, \cdots, N\}$, then a new sub- $\sigma$-field of $\mathbb{C}$ appears. We shall denote it by $\mathcal{E}_{N}$. Note that the conditional expectation $\frac{d \sigma_{h}}{d p}$ with respect to the sub- $\sigma$-field $\mathbb{E}_{N}$ is $\frac{d L_{N} \sigma_{h}}{d L_{N} p}$. So using the martingale convergence theorem we have from (2.15) 


$$
\begin{aligned}
d^{2}\left(\sigma_{h}, \sigma_{g}\right) & =\int_{\Phi^{a}}\left|\sqrt{\frac{d \sigma_{h}}{d p}}\left(\varphi^{a}\right)-\sqrt{\frac{d \sigma_{g}}{d p}}\left(\varphi^{a}\right)\right|^{2} p\left(d \varphi^{a}\right) \\
& =\lim _{n \rightarrow \infty} \int_{R^{n}}\left|\sqrt{\frac{d L_{n} \sigma_{h}}{d L_{n} p}}(y)-\sqrt{\frac{d L_{n} \sigma_{g}}{d L_{n} p}}(y)\right|^{2} d L_{n} p(d y) \\
& =\lim _{n \rightarrow \infty} \int_{R^{n}}\left|\sqrt{\frac{d L_{n} \sigma_{h}}{d y}}(y)-\sqrt{\frac{d L_{n} \sigma_{g}}{d y}}(y)\right|^{2} d y \\
& \leq \lim _{n \rightarrow \infty} \int_{R^{n}} \int_{\left(\Phi_{n}^{*}\right)^{+}}\left|\mathcal{F}\left(q_{n}(\cdot, \xi) T_{n}(h-g)(\cdot, \xi)\right)\right|^{2} q_{n} \mu(d \xi) d y \\
& =\|h-g\|_{2}^{2} .
\end{aligned}
$$

Theorem 2.2. There exists a probability measure $\sigma_{\mu, \theta}$ on $\left(\Phi^{a}, \mho_{\Phi}\right)$ which have the following property.

(P) $\sigma_{\mu, \theta}(E)=0$ if and only if $\sigma_{\mu, \theta, h}(E)=0$ for all $h \in \mathrm{L}_{\mu}^{2}$.

Proof. Take a countable dense set $\left\{h_{1}, \cdots, h_{n}, \cdots\right\} \subset \mathrm{L}_{\mu}^{2}$ and choose positive sequence $\alpha_{n}(n=1, \cdots)$ such that $\sum_{n=1}^{\infty} \alpha_{n}\left\|h_{n}\right\|_{2}^{2}=1$. Then a measure $\sum_{n=1}^{\infty} \alpha_{n} \sigma_{\mu, \theta, h_{n}}$ is a desired one in virtue of the above theorem.

$\sigma_{\mu, \theta}$ is called the spectral measure of the representation $V_{\mu, \theta}$, and sometimes it is simply written as $\sigma$, if no confusion arises.

Theorem 2.3. Let $h, g \in \mathrm{L}_{\mu}^{2}$. Then there exists a complex valued measure $\sigma_{\mu, \theta, h, g} \equiv \sigma_{h, g}$ on $\left(\Phi^{a}, \mathfrak{C}_{\Phi}\right)$ such that

$$
\left\langle V_{\mu, \theta}(\varphi) h, g\right\rangle_{2}=\int_{\Phi^{a}} \exp \left(i\left\langle\varphi, \varphi^{a}\right\rangle\right) \sigma_{h, g}\left(d \varphi^{a}\right),
$$

for all $\varphi \in \Phi$ which satisfies

$$
\begin{gathered}
\sigma_{h, g} \text { is absolutely continuous with } \sigma_{\mu, \theta} \\
\left|\int_{\Phi^{a}} F\left(\varphi^{a}\right) \sigma_{h, g}\left(d \varphi^{a}\right)\right| \leq\|F\|_{\infty, \sigma}\|h\|_{2}\|g\|_{2}
\end{gathered}
$$

for all $F \in \mathrm{L}_{\sigma}^{\infty}$.

Proof. The existence of such $\sigma_{h, g}$ and the absolute continuity is obvious. Next using the same technique in the proof of Theorem 2.1, we have

$$
\frac{d L_{n} \sigma_{h, g}}{d y}=\int_{\left(\Phi_{n}^{*}\right)^{\perp}} \mathcal{F}\left(q_{n}(\cdot, \xi) T_{n} h(\cdot, \xi)\right)(y, \xi) \bar{F}\left(q_{n}(\cdot, \xi) T_{n} g(\cdot, \xi)\right)(y) q_{n} \mu(d \xi) .
$$

It follows that 


$$
\begin{gathered}
\int_{\Phi^{a}}\left|\frac{d \sigma_{h, g}}{d \sigma_{\mu, \theta}}\left(\varphi^{a}\right)\right| \sigma_{\mu, \theta}\left(d \varphi^{a}\right)=\lim _{n \rightarrow \infty} \int_{R^{n}}\left|\frac{d L_{n} \sigma_{h, g}}{d L_{n} \sigma_{\mu, \theta}}(y)\right| L_{n} \sigma_{\mu, \theta}(d y) \\
=\lim _{n \rightarrow \infty} \int_{R^{n}}\left|\frac{d L_{n} \sigma_{h, g}}{d y}\right| d y \\
\leq \underline{\lim }_{n \rightarrow \infty} \int_{R^{n}}\left\{\int_{\left(\Phi_{n}^{*}\right)^{\perp}}\left|\mathcal{F}\left(q_{n}(\cdot, \xi) T_{n} h(\cdot, \xi)\right)\right|^{2} q_{n} \mu(d \xi)\right\}^{1 / 2} . \\
\quad\left\{\int_{\left(\Phi_{n}^{*}\right)^{+}}\left|\mathcal{F}\left(q_{n}(\cdot, \xi) T_{n} g(\cdot, \xi)\right)\right|^{2} q_{n} \mu(d \xi)\right\}^{1 / 2} d y \leq\|h\|_{2}\|g\|_{2 .}
\end{gathered}
$$

(2.17) is a direct consequence of the above inequality.

By virtue of the above theorem, we can define a bounded operator $T_{\mu, \theta}(F) \equiv$ $T(F)$ on $\mathrm{L}_{\mu}^{2}$ for each $F \in \mathrm{L}_{\sigma}^{\infty}$ such that

$$
\langle T(F) h, g\rangle_{2}:=\int_{\Phi^{a}} F\left(\varphi^{a}\right) \sigma_{\mu, \theta, h, g}\left(d \varphi^{a}\right),
$$

for all $h, g \in \mathrm{L}_{\mu}^{2}$.

Proposition 2.1. $T(F)$ has the following properties.

$$
\begin{gathered}
T(F)^{*}=T(\bar{F}) \\
T(F \cdot G)=T(F) \cdot T(G) \\
T \text { is injective and }\|T(F)\|_{o p}=\|F\|_{\infty} .
\end{gathered}
$$

Proof. As for (2.20),

$$
\begin{aligned}
\widehat{\sigma_{g, h}}(\varphi) & =\left\langle V_{\mu, \theta}(\varphi) g, h\right\rangle_{2}=\overline{\left\langle V_{\mu, \theta}(-\varphi) h, g\right\rangle_{2}} \\
& =\int_{\Phi^{a}} \exp \left(i\left\langle\varphi, \varphi^{a}\right\rangle\right) \overline{\sigma_{h, g}}\left(d \varphi^{a}\right)=\widehat{\sigma_{h, g}}(\varphi) .
\end{aligned}
$$

So we have $\sigma_{g, h}=\overline{\sigma_{h, g}}$. It follows that

$$
\begin{aligned}
\left\langle T(F)^{*} h, g\right\rangle_{2} & =\overline{\int_{\Phi^{a}} F\left(\varphi^{a}\right) \sigma_{g, h}\left(d \varphi^{a}\right)} \\
& =\int_{\Phi^{a}} \bar{F}\left(\varphi^{a}\right) \sigma_{h, g}\left(d \varphi^{a}\right)=\langle T(\bar{F}) h, g\rangle_{2} .
\end{aligned}
$$

We shall prove (2.21). It is easy to see that the equality holds in the case $F\left(\varphi^{a}\right)$ $=\exp \left(i\left\langle\varphi_{1}, \varphi^{a}\right\rangle\right)$ and $G\left(\varphi^{a}\right)=\exp \left(i\left\langle\varphi_{2}, \varphi^{a}\right\rangle\right)$. Next we shall substitute the indicator function $\chi_{E}$ of a measurable set $E \in \mathbb{C}$ for $\exp \left(i\left\langle\varphi_{1}, \varphi^{a}\right\rangle\right)$. Then $\left\langle T\left(\chi_{E} \cdot G\right) h, g\right\rangle_{2}$ and $\left\langle T\left(\chi_{E}\right) T(G) h, g\right\rangle_{2}$ are both regarded as complex valued measures on $\Subset$ and their characteristic functions coincide by the first step. Thus they are equal to each other. Lastly we substitute $\chi_{F}, F \in \mathbb{C}$ for $G=\exp \left(i<\varphi_{2}\right.$, $\left.\left.\varphi^{a}\right\rangle\right)$ and repeat the same argument as above. So (2.21) certainly holds in the 
case that $F$ and $G$ are step functions. The general case easily follows from (2.17).

As for (2.22), if we have $T(F)=0$ for some $F \in \mathrm{L}_{\sigma}^{\infty}$, then we may assume that $F\left(\varphi^{a}\right) \geq 0$ for $\sigma$-a.e. $\varphi^{a}$, because $T\left(|F|^{2}\right)=T(F) T(\bar{F})=0$. Since $\int_{\Phi^{a}} F\left(\varphi^{a}\right)$ $\sigma_{h}\left(d \varphi^{a}\right)=0$ for all $h \in \mathrm{L}_{\mu}^{2}, F\left(\varphi^{a}\right)=0$ for $\sigma_{h}$-a.e. $\varphi^{a}$, which is equivalent to $F\left(\varphi^{a}\right)$ $=0$ for $\sigma_{\mu, \theta}$-a.e. $\varphi^{a}$.

The norm equality is a direct consequence of $*$-isomorphism $T_{\mu, \theta}$. (For example see p5 in [8] or refer the later discussions.)

2.2 Decomposition of $V_{\mu, \theta}$ by a direct integral. We shall start at the following equality,

$$
\langle T(F) h, g\rangle_{2}=\int_{\Phi^{a}} F\left(\varphi^{a}\right) \frac{d \sigma_{h, g}}{d \sigma}\left(\varphi^{a}\right) \sigma\left(d \varphi^{a}\right),
$$

and follow after J. Diximier [1]. First we shall take and fix a dense $\mathbf{Q}$-linear subspace $V \subset \mathrm{L}_{\mu}^{2}$ whose cardinal is countable. Then there exists a $\sigma$-negligible set $N$ such that for every $\varphi^{a} \in N^{c}$, the function $\left(v, v^{\prime}\right) \longmapsto \frac{d \sigma_{v, v^{\prime}}}{d \sigma}\left(\varphi^{a}\right)$ is a positive definite sesquilinear form on $V$. Let $H_{\mu, \theta}\left(\varphi^{a}\right) \equiv H\left(\varphi^{a}\right)$ be the Hilbert space obtained from $V$ by passing to the quotient and completing with this sesquilinear form. We put $H\left(\varphi^{a}\right)=\{0\}$ for $\varphi^{a} \in N$ and denote the image of $v_{n}$ by the canonical map $V \longmapsto H\left(\varphi^{a}\right)$ by $v_{n}\left(\varphi^{a}\right)$. Then

$$
\frac{d \sigma_{v_{n}, v_{m}}}{d \sigma}\left(\varphi^{a}\right)=\left\langle v_{n}\left(\varphi^{a}\right) \mid v_{m}\left(\varphi^{a}\right)\right\rangle_{H\left(\varphi^{a}\right)} \text {, if } \varphi^{a} \in N^{c} \text { and }=0 \text { otherwise }
$$

So they are $\widetilde{C}_{\Phi}$-measurable. Further $\left\{v_{n}\left(\varphi^{a}\right)\right\}_{n}$ forms a total sequence $\Longleftrightarrow$ spans a dense linear subspace) in each $H\left(\varphi^{a}\right)$. Consequently there exists exactly one measurable structure $R_{\mu, \theta} \equiv R$ on the $H\left(\varphi^{a}\right)$ 's with which every field, $\varphi^{a}$ $\longmapsto v_{n}\left(\varphi^{a}\right)(n=1, \cdots$,$) is measurable. (See, p167 in [1].) Hence a direct$ integral $H:=\int^{\oplus} H\left(\varphi^{a}\right) \sigma\left(d \varphi^{a}\right)$ has been constructed with the spectral measure $\sigma$. We note that

$$
\operatorname{dim} H\left(\varphi^{a}\right) \geq 1
$$

for $\sigma$-a.e. $\varphi^{a}$. Because $K:=\left\{\varphi^{a} \mid \operatorname{dim} H\left(\varphi^{a}\right)=0\right\} \ni \varphi^{a}$ is equivalent to $\frac{d \sigma_{v_{n}, v_{m}}}{d \sigma}\left(\varphi^{a}\right)$ $=0$ for all $n, m$ from which it follows that $\sigma_{v_{n}, v_{m}}(K)=0$. As $\left\{v_{n}\right\}_{n}$ is dense, so we have $\sigma(K)=0$ by virtue of (2.7).

Theorem 2.4. (1) $\mathrm{L}_{\mu}^{2}$ is isomorphic to $H$ by a unitary map $S_{\mu, \theta} \equiv S$ such that 


$$
S T(F)=T^{H}(F) S
$$

for all $F \in \mathrm{L}_{\sigma}^{\infty}$, where $T^{H}(F): \eta\left(\varphi^{a}\right) \in H \longmapsto F\left(\varphi^{a}\right) \eta\left(\varphi^{a}\right) \in H$ is the diagonalisable operator.

(2) If $\mathrm{L}_{\mu}^{2}$ is isomorphic to another direct integral $H^{\prime}=\int^{\oplus} H^{\prime}\left(\varphi^{a}\right) \sigma^{\prime}\left(d \varphi^{a}\right)$ with another spectral measure $\sigma^{\prime}$ by a map $S^{\prime}$ which have the same property as (2.25), then $\operatorname{dim} H\left(\varphi^{a}\right)=\operatorname{dim} H^{\prime}\left(\varphi^{a}\right)$ for $\sigma$-a.e. $\varphi^{a}$.

Proof. For (1), let $F_{n}(n=1, \cdots, N) \in \mathrm{L}_{\sigma}^{\infty}$. Then

$$
\begin{aligned}
\left\|\sum_{n=1}^{N} T\left(F_{n}\right) v_{n}\right\|_{2}^{2} & =\sum_{n, m=1}^{N} \int_{\Phi^{a}} F_{n}\left(\varphi^{a}\right) \bar{F}_{m}\left(\varphi^{a}\right) \frac{d \sigma_{n, v m}}{d \sigma}\left(\varphi^{a}\right) \sigma\left(d \varphi^{a}\right) \\
& =\sum_{n, m=1}^{N} \int_{\Phi^{a}} F_{n}\left(\varphi^{a}\right) \bar{F}_{m}\left(\varphi^{a}\right)\left\langle v_{n}\left(\varphi^{a}\right) \mid v_{m}\left(\varphi^{a}\right)\right\rangle_{H\left(\varphi^{a}\right)} \sigma\left(d \varphi^{a}\right) \\
& =\int_{\Phi^{a}}\left\|\sum_{n=1}^{N} F_{n}\left(\varphi^{a}\right) v_{n}\left(\varphi^{a}\right)\right\|_{H\left(\varphi^{a}\right)}^{2} \sigma\left(d \varphi^{a}\right) .
\end{aligned}
$$

Therefore a map $S: \sum_{n=1}^{N} T\left(F_{n}\right) v_{n} \longmapsto \sum_{n=1}^{N} F_{n}\left(\varphi^{a}\right) v_{n}\left(\varphi^{a}\right)$ is well defined and is extended to a unitary map from $\mathrm{L}_{\mu}^{2}$ to $H$. That the image of $S$ is dense follows from the totality of $\left\{v_{n}\right\}_{n}$.

For (2), from the assumption, $S^{\prime} S^{-1}$ is a decomposable operator. That is, $S^{\prime} S^{-1}$ induces an isomorphic operator $\Sigma\left(\varphi^{a}\right): H\left(\varphi^{a}\right) \longmapsto H^{\prime}\left(\varphi^{a}\right)$ for $\lambda$-a.e. $\varphi^{a}$. (See, p187 in [1].) Thus the corresponding dimensions are the same one.

Example 1. $\boldsymbol{R}^{n}$-quasi-invariant measure $\mu$ on $\left(\boldsymbol{R}^{n}, \mathfrak{B}\left(\boldsymbol{R}^{n}\right)\right)$. That is, $\mu(d x)$ $=\rho(x) d x, \rho(x)>0$ for $\lambda_{n}$-a.e. $x$.

In this case, as we have seen in (2.14), for any 1-cocycle $\theta(x, \varphi)$ there exists some measurable function $q(x)$ with $|q(x)|=1$ such that for each $\varphi \in \boldsymbol{R}^{n}, \theta(x, \varphi)=$ $q(x-\varphi) q(x)^{-1}$ for a.e. $x \in \boldsymbol{R}^{n}$.

$$
\begin{aligned}
\left\langle V_{\mu, q}(\varphi) h, g\right\rangle_{2} & =\int_{\boldsymbol{R}^{n}} \sqrt{\frac{d \mu_{\varphi}}{d \mu}}(x) q(x-\varphi) h(x-\varphi) \bar{q}(x) \bar{g}(x) \mu(d x) \\
& =\int_{\boldsymbol{R}^{n}} \sqrt{\rho(x-\varphi)} q(x-\varphi) h(x-\varphi) \sqrt{\rho(x)} \bar{q}(x) \bar{g}(x) d x \\
& =\int_{\boldsymbol{R}^{n}} \exp (i\langle\varphi, y\rangle) \mathcal{F}(q \sqrt{\rho} h)(y) \bar{F}(q \sqrt{\rho} g)(y) d y .
\end{aligned}
$$

Thus the unitary map $S_{q}$ is $\rho(y)^{-1 / 2} \mathscr{F}(q \sqrt{\rho} h)(y)$, the spectral measure is $\mu$, and $H_{q}(y)=\boldsymbol{R}$.

Theorem 2.5. Let $\mu$ and $\mu^{\prime}$ be $\Phi$-quasi-invariant probability measures on $(X, \mathfrak{B})$, and $\theta$ and $\theta^{\prime}$ be 1-cocycles. Then $\left(V_{\mu, \theta}, \Phi\right)$ is equivalent to $\left(V_{\mu^{\prime}, \theta^{\prime}}, \Phi\right)$, if and only if $\sigma_{\mu, \theta} \cong \sigma_{\mu^{\prime}, \theta^{\prime}}$ and $\operatorname{dim} H_{\mu, \theta}\left(\varphi^{a}\right)=\operatorname{dim} H_{\mu^{\prime}, \theta^{\prime}}\left(\varphi^{a}\right)$ for $\sigma_{\mu, \theta}-$ a.e. $\varphi^{a}$. 
Proof. For the proof, we only have to note that an operator which commutes with every diagonalisable operator is decomposable.

Theorem 2.6. Let $\lambda$ be any finite measure on $\left(\Phi^{a}, \bigodot_{\Phi}\right)$ which is absolutely continuous with $\sigma_{\mu, \theta}$. Then there exists some $h \in \mathrm{L}_{\mu}^{2}$ such that $\sigma_{\mu, \theta, h}=\lambda$.

Proof. We shall take $\eta \in \int^{\oplus} H_{\mu, \theta}\left(\varphi^{a}\right) \sigma_{\mu, \theta}\left(d \varphi^{a}\right)$ such that $\left\|\eta\left(\varphi^{a}\right)\right\|=1$ for $\lambda$-a.e. $\varphi^{a}$. The existence of such $\eta$ is assured by (2.24). (Also see, p162 in [1].) Then an element $h$ defined by $h\left(\varphi^{a}\right)=S^{-1}\left(\sqrt{\frac{d \lambda}{d \sigma}} \eta\right)$ is a desired one.

\section{§3. Lifting and Restriction of Canonical Representations}

3.1 Dense case. As before let $\mu$ be a probability measure on $(X, \mathfrak{B})$ and put

$$
A_{\mu}^{\circ}:=\left\{\varphi \in X \mid \mu_{t \varphi} \cong \mu \text { for all } t \in \mathbb{R}\right\},
$$

which is the maximal linear space consists of admissible shifts of $\mu$. We give a metric $d_{\mu}$ on $A_{\mu}^{\circ}$ such that

$$
d_{\mu}\left(\varphi_{1}, \varphi_{2}\right):=\sup _{|t| \leq 1}\left\|\sqrt{\frac{d \mu_{t \varphi_{1}}}{d \mu}}-\sqrt{\frac{d \mu_{t \varphi_{2}}}{d \mu}}\right\|_{2}
$$

It is well known that $d_{\mu}$ has the following properties. (See, [9].)

(P.1) $\quad d_{\mu}$ is translationally invariant and $\left(A_{\mu}^{\circ}, d_{\mu}\right)$ is separable. (The separability is a consequence of the assumption that $\mathrm{L}_{\mu}^{2}$ is separable.)

(P.2) $\varphi \rightarrow 0$ in $d_{\mu}$ if and only if $\left\|\mu_{t \varphi}-\mu\right\|_{\text {tot }} \rightarrow 0$ for any fixed $t \in \mathbb{R}$.

(P.3) The topology derived from $d_{\mu}$ is stronger than the weak topology $\sigma(X$, $\left.X^{*}\right)$.

(P.4) If $\left\{\varphi_{n}\right\}_{n}$ is a Cauchy sequence in $d_{\mu}$, and $\varphi_{n} \rightarrow \varphi$ in $\sigma\left(X, X^{*}\right)$, then $\varphi_{n} \rightarrow \varphi$ in $d_{\mu}$.

(P.5) $\varphi \rightarrow 0$ in $d_{\mu}$, if and only if $V(t \varphi) \rightarrow \mathrm{I}$ in the strong operator topology for any fixed $t \in \mathbb{R}$.

Proposition 3.1. Let $\Phi$ be a linear subspace of $A_{\mu}^{\circ}$ and $\theta(x, \varphi)$ be a 1 -cocycle defined for $\varphi \in \Phi$. Then $\varphi \rightarrow 0$ in $d_{\mu}$ if and only if $\langle\varphi, \cdot\rangle \rightarrow 0$ in $\sigma_{\mu, \theta}$.

Proof. $(\Rightarrow)$ From the assumption and (P.5), we have $<V_{\mu, \theta}(t \varphi) h$, $h\rangle_{2} \longrightarrow\|h\|_{2}^{2}$ for each fixed $h \in \mathrm{L}_{\mu}^{2}$. Especially,

$\int_{\Phi^{a}} \exp \left(i t\left\langle\varphi, \varphi^{a}\right\rangle\right) \sigma_{\mu, \theta}\left(d \varphi^{a}\right) \rightarrow 1$ for each fixed $t \in \boldsymbol{R}$, which is equivalent to 
$\langle\varphi, \cdot\rangle \rightarrow 0$ in $\sigma_{\mu, \theta}$

$(\Leftarrow)$ Since $\langle\varphi, \cdot\rangle$ converges to 0 in $\sigma_{\mu, \theta, 1}$, so

$$
\left\langle V_{\mu, \theta}(t \varphi) 1,1\right\rangle_{2}=\int_{\Phi^{a}} \exp \left(i\left\langle\varphi, \varphi^{a}\right\rangle\right) \sigma_{\mu, \theta, 1}\left(d \varphi^{a}\right) \rightarrow 1
$$

for each fixed $t \in \boldsymbol{R}$. Thus we have

$$
\begin{aligned}
\int_{X}\left\{1-\sqrt{\frac{d \mu_{t \varphi_{n}}}{d \mu}}(x)\right\}^{2} \mu(d x)= & 2\left\{1-\int_{X} \sqrt{\frac{d \mu_{t \varphi_{n}}}{d \mu}}(x) \mu(d x)\right\} \\
& \leq 2\left\{1-\operatorname{Re}\left\langle V_{\mu, \theta}(t \varphi) 1,1\right\rangle\right\} \rightarrow 0 .
\end{aligned}
$$

Now let $\Phi$ and $\Psi$ be two linear subspaces of $A_{\mu}^{\circ}$ such that $\Phi \subseteq \Psi \subseteq A_{\mu}^{\circ}$ and $\iota$ be the imbedding map from $\Phi$ to $\Psi$. We shall denote the corresponding operator $T_{\mu, \theta}(\cdot)$ to $\Phi$ and $\Psi$ by $T_{\mu, \theta}^{\Phi}(\cdot)$ and $T_{\mu, \theta}^{\Psi}(\cdot)$, respectively. Then it is easily checked that

$$
\left\langle T_{\mu, \theta}^{\Phi}(E) h, g\right\rangle_{2}=\left\langle T_{\mu, \theta}^{\Psi}\left(\left({ }^{t} \iota\right)^{-1}(E)\right) h, g\right\rangle_{2}
$$

for all $E \in \mathfrak{C}_{\Phi}$. Hence the spectral measure can be chosen as

$$
\sigma_{\mu, \theta}^{\Phi}={ }^{t} \iota \sigma_{\mu, \theta}^{\Psi}
$$

Lemma 3.1. If $\Phi$ is dense in $\Psi$ with the derived topology from $d_{\mu}$, then $\mathfrak{c}_{\Psi}=\left({ }^{t} \iota\right)^{-1}\left(\mathfrak{\complement}_{\Phi}\right) \bmod \sigma_{\mu, \theta}^{\Psi}$.

Proof. Put

$$
\text { 厄: }=\left\{\left.E \in \mho_{\Psi}\right|^{\Xi} E_{1} \in \mho_{\Phi} \text { s.t., } E=\left({ }^{t} \iota\right)^{-1}\left(E_{1}\right) \bmod \sigma_{\mu, \theta}^{\Psi}\right\} .
$$

Then $\Subset$ is a $\sigma$-field. Moreover for any fixed $\psi \in \Psi,\left\langle\psi, \psi^{a}\right\rangle$ is equal to a (-measurable function for $\sigma_{\mu, \theta}^{\Psi}$-a.e. $\psi^{a}$, because $\left\langle\varphi_{n}, \psi^{a}\right\rangle$ converges to $\left\langle\psi, \psi^{a}\right\rangle$ in $\sigma_{\mu, \theta}^{\Psi}$, where $\left\{\varphi_{n}\right\}_{n}$ is any sequence of $\Phi$ such that $d_{\mu}\left(\varphi_{n}, \psi\right) \rightarrow 0(n \rightarrow \infty)$.

Under the same assumption of Lemma 3.1 we also see that for any $F \in \mathrm{L}_{\sigma, u, \theta}^{\infty}$, there exists $f \in \mathrm{L}_{\sigma_{\mu, \theta}^{\Phi}}^{\infty}$ such that $F\left(\psi^{a}\right)=f\left({ }^{t} \iota \psi^{a}\right)$ for $\sigma_{\mu, \theta}^{\Psi}$-a.e. $\psi^{a}$.

Theorem 3.1. Let $\Phi \subseteq \Psi \subseteq A_{\mu}^{\circ}$ and $\Phi$ be dense in $\Psi$. If an expression of the direct integral for $\left(V_{\mu, \theta}, \Phi\right)$ is $\int^{\oplus} H_{\mu, \theta}^{\Phi}\left(\varphi^{a}\right) \sigma_{\mu, \theta}^{\Phi}\left(d \varphi^{a}\right)$, then $\int^{\oplus} H_{\mu, \theta}^{\Phi}\left({ }^{t} \iota \psi^{a}\right)$ $\sigma_{\mu, \theta}^{\Psi}\left(d \psi^{a}\right)$ is an expression of the direct integral for $\left(V_{\mu, \theta}, \Psi\right)$.

Proof. For the proof it is enough to show that $\operatorname{dim} H_{\mu, \theta}^{\Phi}\left({ }^{t} \iota \psi^{a}\right)=\operatorname{dim} H_{\mu, \theta}^{\Psi}$ $\left(\psi^{a}\right)$ for $\sigma_{\mu, \theta}^{\psi}$-a.e. $\psi^{a}$.

Now take $F \in \mathrm{L}_{\sigma_{\mu, \theta}^{\Psi}}^{\infty}$ and $h, g \in \mathrm{L}_{\mu}^{2}$. Then 


$$
\left\langle T_{\mu, \theta}^{\Psi}(F) h, g\right\rangle_{2}=\left\langle T_{\mu, \theta}^{\Psi}\left(f \circ{ }^{t} \iota\right) h, g\right\rangle_{2}=\left\langle T_{\mu, \theta}^{\Phi}(f) h, g\right\rangle_{2} .
$$

Thus,

$$
\begin{aligned}
& \int_{\Psi^{a}} F\left(\phi^{a}\right)\left\langle S_{\mu, \theta}^{\Psi} h \mid S_{\mu, \theta}^{\Psi} g\right\rangle\left(\psi^{a}\right) \sigma_{\mu, \theta}^{\Psi}(\left.d \psi^{a}\right) \\
&=\int_{\Psi^{a}} F\left(\psi^{a}\right)\left\langle S_{\mu, \theta}^{\Phi} h \mid S_{\mu, \theta}^{\Phi} g\right\rangle\left({ }^{t} \iota \psi^{a}\right) \sigma_{\mu, \theta}^{\Psi}\left(d \psi^{a}\right) .
\end{aligned}
$$

Here we shall choose a sequence $\left\{h_{n}\right\}_{n} \subset \mathrm{L}_{\mu}^{2}$ which satisfies $\left\{S_{\mu, \theta}^{\Psi} h_{n}\left(\psi^{a}\right)\right\}_{n}$ and $\left\{S_{\mu, \theta}^{\Phi} h_{n}\left(\varphi^{a}\right)\right\}_{n}$ form total sequence at every $H_{\mu, \theta}^{\Psi}\left(\psi^{a}\right)$ and $H_{\mu, \theta}^{\Phi}\left(\varphi^{a}\right)$, respectively. Then it follows from (3.3) that for all $N$ and for all $q_{n} \in \mathbb{Q},\left\|\sum_{n=1}^{N} q_{n} S_{\mu, \theta}^{\Psi} h_{n}\left(\psi^{a}\right)\right\|$ $=\left\|\sum_{n=1}^{N} q_{n} S_{\mu, \theta}^{\Phi} h_{n}\left({ }^{t} \iota \psi^{a}\right)\right\|$ for $\sigma_{\mu, \theta}^{\Psi}$-a.e. $\psi^{a}$. Therefore a map $S_{\mu, \theta}^{\Psi} h_{n}\left(\psi^{a}\right) \longmapsto S_{\mu, \theta}^{\Phi}$ $h_{n}\left({ }^{t} \iota \psi^{a}\right)$ is extended to a unitary map from $H_{\mu, \theta}^{\Psi}\left(\psi^{a}\right)$ to $H_{\mu, \theta}^{\Phi}\left({ }^{t} \iota \psi^{a}\right)$.

Theorem 3.2. Let $\mu$ and $\mu^{\prime}$ be probability measures on $(X, \mathfrak{B}), \Phi, \Psi$ be linear subspaces of $X$ such that $\Phi \subseteq \Psi \subseteq A_{\mu}^{\circ} \cap A_{\mu^{\prime}}^{\circ}$, and $\theta, \theta^{\prime}$ be 1-cocycles defined on $X \times \Psi$.

(1) If $\sigma_{\mu, \theta}^{\Phi} \cong \sigma_{\mu^{\prime}, \theta^{\prime}}^{\Phi}$ and $\Phi$ is dense in $\Psi$ with either $d_{\mu}$ or $d_{\mu^{\prime}}$, then it also holds with another one and $\sigma_{\mu, \theta}^{\Psi} \cong \sigma_{\mu^{\prime}, \theta^{\prime}}^{\Psi}$.

(2) If $\left(V_{\mu, \theta}, \Phi\right) \cong\left(V_{\mu^{\prime}, \theta^{\prime}}, \Phi\right)$ and $\Phi$ is dense in $\Psi$ with either $d_{\mu}$ or $d_{\mu^{\prime}}$, then it also holds that $\left(V_{\mu, \theta}, \Psi\right) \cong\left(V_{\mu^{\prime}, \theta^{\prime}}, \Psi\right)$.

Proof. (1). Assume that $\Phi$ is dense in $\Psi$. Then for any $\psi \in \Psi$, there exists a sequence $\left\{\varphi_{n}\right\}_{n} \subset \Phi$ such that $d_{\mu}\left(\varphi_{n}, \psi\right) \rightarrow 0$ and thus $\varphi_{n} \rightarrow \psi$ in $\sigma\left(X, X^{*}\right)$. It follows from the assumption and from Proposition 3.1 that $\left\{\varphi_{n}\right\}_{n}$ is also a Cauchy sequence in $d_{\mu^{\prime}}$. Therefore they converge to $\phi$ by virtue of (P.4).

As for the second half of (a), repeating the same work in the proof of Lemma 3.1 we see that for any $E \in \bigodot{ }_{\Psi}$ there exists $E_{1} \in \mathfrak{C}_{\Phi}$ such that $\sigma_{\mu, \theta}^{\Psi}\left(E \Theta\left({ }^{t} \iota\right)^{-1}\left(E_{1}\right)\right)$ $=\sigma_{\mu^{\prime}, \theta^{\prime}}^{\Psi}\left(E \ominus\left({ }^{t} \iota\right)^{-1}\left(E_{1}\right)\right)=0$. The equivalence of measures immediately follows from this matter.

For (2), it is a direct consequence of (1) in the above theorem and Theorem 3.1.

3.2 General case. Let $\Phi$ and $\Psi$ be two linear subspaces of $A_{\mu}^{\circ}$ such that $\Phi \subseteq \Psi$. We shall examine how the expression of the direct integral for $\left(V_{\mu, \theta}, \Psi\right)$ derives it for $\left(V_{\mu, \theta}, \Phi\right)$. So let us take linearly independent sets $\left\{\varphi_{n}\right\}_{n}$ and $\left\{\varphi_{n}^{\prime}\right\}_{n}$ such that $\varphi_{n}(n=1, \cdots)$ span a dense linear subspace $\Phi_{0}$ of $\Phi$, and both $\varphi_{n}$ and $\varphi_{n}^{\prime}(n=1, \cdots)$ span a dense linear subspace $\Psi_{0}$ of $\Psi$. From what we have seen in 3.1, it is essential to observe the direct integrals for $\left(V_{\mu, \theta}, \Phi_{0}\right)$ and $\left(V_{\mu, \theta}, \Psi_{0}\right)$ and to discuss their mutual relation for this problem. An advantage of changing the spaces into new ones is that $\left(\Psi_{0}, \widetilde{C}_{\Psi_{0}}\right)$ is Borel isomorphic to the natural measurable space $\left(\boldsymbol{R}^{\infty}, \mathfrak{B}\left(\boldsymbol{R}^{\infty}\right)\right)$ of the countable direct product of $\mathbb{R}$ and there- 
fore $\left(\Psi_{0}^{a}, \mathcal{C}_{\Psi_{0}}\right)$ is a standard space. In particular, $\sigma_{\mu, \theta}^{\Psi_{0}}$ is decomposed to regular conditional probabilities $Q_{\mu, \theta}^{\varphi_{0}^{a}}$ given ${ }^{t} c_{0}=\varphi_{0}^{a}$ as follows, where $\iota_{0}$ is the imbedding map from $\Phi_{0}$ to $\Psi_{0}$.

$$
\sigma_{\mu, \theta}^{\Psi_{0}}\left(\left({ }^{t} c_{0}\right)^{-1}(E) \cap F\right)=\int_{E} Q_{\mu, \theta}^{\varphi_{0}^{a}}(F) \sigma_{\mu, \theta}^{\Phi_{0}}\left(d \varphi_{0}^{a}\right)
$$

for all $E \in \bigodot_{\Phi_{0}}$, where $\sigma_{\mu, \theta}^{\Phi_{0}}={ }^{t} \iota_{0} \sigma_{\mu, \theta}^{\Psi_{0}}$. Let $R^{\Psi_{0}}$ be the measurable field used to define the direct integral $\int^{\oplus} H_{\mu, \theta}^{\Psi_{0}}\left(\psi_{0}^{a}\right) \sigma_{\mu, \theta}^{\Psi_{0}}\left(d \psi_{0}^{a}\right)$ and put

$$
H_{\mu, \theta}^{\Phi_{0}}\left(\varphi_{0}^{a}\right):=\left\{\eta \in R^{\Psi_{0}} \mid \int_{\Psi_{0}^{a}}\left\|\eta\left(\psi_{0}^{a}\right)\right\|^{2} Q_{\mu, \theta}^{\varphi_{0}^{a}}\left(d \psi_{0}^{a}\right)<\infty\right\} .
$$

In order to assign a measurable field with respect to $\left\{H_{\mu, \theta}^{\Phi_{0}}\left(\varphi_{0}^{a}\right)\right\}$, we shall take $\left\{\eta_{n}\right\}_{n}$ which satisfies

(P.6) if $\operatorname{dim} H_{\mu, \theta}^{\Psi_{0}}\left(\psi_{0}^{a}\right)=\infty, \eta_{1}\left(\psi_{0}^{a}\right), \cdots, \eta_{n}\left(\psi_{0}^{a}\right), \cdots$ is a c.o.n.s.in $H_{\mu, \theta}^{\Psi_{0}}\left(\psi_{0}^{a}\right)$,

(P.7) if $\operatorname{dim} H_{\mu, \theta}^{\Psi_{0}}\left(\psi_{0}^{a}\right)=d<\infty, \eta_{1}\left(\psi_{0}^{a}\right), \cdots, \eta_{d}\left(\psi_{0}^{a}\right)$ is a c.o.n.s.in $H_{\mu, \theta}^{\psi_{0}}\left(\psi_{0}^{a}\right)$ and $\eta_{n}\left(\psi_{0}^{a}\right)=0$ for $n>d$.

(For the existence of such $\left\{\eta_{n}\right\}_{n}$ see p166 in [1].)

Further we shall take a countable algebra $\mathscr{F}_{0}=\left\{F_{n}\right\}_{n}$ which generates $\mathfrak{S}_{\Psi_{0}}$. Then $\chi_{F_{p}} \cdot \eta_{n}(n, p=1, \cdots)$ belongs to $H_{\mu, \theta}^{\Phi_{0}}\left(\varphi_{0}^{a}\right)$ and forms a total sequence in $H_{\mu, \theta}^{\Phi_{0}}\left(\varphi_{0}^{a}\right)$, as is easily seen. Besides,

$$
\left\langle\chi_{F_{p}} \cdot \eta_{n} \mid \chi_{F_{q}} \cdot \eta_{m}\right\rangle\left(\varphi_{0}^{a}\right)=\delta_{n, m} Q_{\mu, \theta}^{\varphi_{0}^{a}}\left(F_{p} \cap F_{q}\right)
$$

is $\widetilde{S}_{\Phi_{0}}$-measurable. Thus there exists a unique measurable field $R^{\Phi_{0}}$ with which all the $\chi_{F_{p}} \cdot \eta_{n}$ are measurable, (See, p167 in [1].) and a direct integral $\int^{\oplus} H_{\mu, \theta}^{\Phi_{0}}$ $\left(\varphi_{0}^{a}\right) \sigma_{\mu, \theta}^{\Phi_{0}}\left(d \varphi_{0}^{a}\right)$ is defined with the measure $\sigma_{\mu, \theta}^{\Phi_{0}}$.

Theorem 3.3. An expression of the direct integral $\left(V_{\mu, \theta}, \Phi\right)$ is $\int^{\oplus} H_{\mu, \theta}^{\Phi_{0}}$ $\left({ }^{t} J \varphi^{a}\right) \sigma_{\mu, \theta}^{\Phi}\left(d \varphi^{a}\right)$, where $J$ is the imbedding map from $\Phi_{0}$ to $\Phi$.

Proof. By Theorem 3.1, it is enough to show that $\mathrm{L}_{\mu}^{2}$ is canonically isomorphic to $\int^{\oplus} H_{\mu, \theta}^{\Phi_{0}}\left(\varphi_{0}^{a}\right) \sigma_{\mu, \theta}^{\Phi_{0}}\left(d \varphi_{0}^{a}\right)$. For this we put $h_{n}:=\left(S_{\mu, \theta}^{\Psi_{0}}\right)^{-1} \eta_{n}$ and take $f \in \mathrm{L}_{\sigma_{\mu, \theta}^{\Phi_{0}}}^{\infty}$ and $F_{n}, F_{n}^{\prime} \in \mathrm{L}_{\sigma_{\mu, \theta}^{\Psi} \psi_{0}}^{\infty}\left(n, n^{\prime}=1, \cdots, N\right)$. Then

$$
\begin{aligned}
& \left\langle T_{\mu, \theta}^{\Phi_{0}}(f)\left(\sum_{n=1}^{N} T_{\mu, \theta}^{\Psi_{0}}\left(F_{n}\right) h_{n}\right), \sum_{n=1}^{N} T_{\mu, \theta}^{\Psi_{0}}\left(F_{n}^{\prime}\right) h_{n}\right\rangle_{2} \\
& =\left\langle T_{\mu, \theta}^{\Psi_{0}}\left(f^{\circ}{ }^{t} \iota_{0}\right)\left(\sum_{n=1}^{N} T_{\mu, \theta}^{\Psi_{0}}\left(F_{n}\right) h_{n}\right), \sum_{n=1}^{N} T_{\mu, \theta}^{\Psi_{0}}\left(F_{n}^{\prime}\right) h_{n}\right\rangle_{2}
\end{aligned}
$$




$$
\begin{aligned}
& =\sum_{n, m=1}^{N} \int_{\Psi_{0}^{a}} f\left({ }^{t} c_{0} \psi_{0}^{a}\right) F_{n}\left(\psi_{0}^{a}\right) \overline{F_{m}^{\prime}}\left(\psi_{0}^{a}\right)\left\langle\eta_{n} \mid \eta_{m}\right\rangle\left(\psi_{0}^{a}\right) \sigma_{\mu, \theta}^{\Psi_{0}}\left(d \psi_{0}^{a}\right) \\
& =\sum_{n, m=1}^{N} \int_{\Phi_{0}^{a}} f\left(\varphi_{0}^{a}\right) \sigma_{\mu, \theta}^{\Phi_{0}}\left(d \varphi_{0}^{a}\right) \int_{\psi_{0}^{a}} F_{n}\left(\psi_{0}^{a}\right) \overline{F_{m}^{\prime}}\left(\psi_{0}^{a}\right)\left\langle\eta_{n} \mid \eta_{m}\right\rangle\left(\psi_{0}^{a}\right) Q_{\mu, \theta}^{\varphi_{0}^{a}}\left(d \psi_{0}^{a}\right) \\
& =\int_{\Phi_{0}^{a}} f\left(\varphi_{0}^{a}\right)\left\langle\sum_{n=1}^{N} F_{n} \cdot \eta_{n} \mid \sum_{n=1}^{N} F_{n}^{\prime} \cdot \eta_{n}\right\rangle\left(\varphi_{0}^{a}\right) \sigma_{\mu, \theta}^{\Phi_{0}}\left(d \varphi_{0}^{a}\right) .
\end{aligned}
$$

So a map $S_{\mu, \theta}^{\Phi_{0}}: \sum_{n=1}^{N} T_{\mu, \theta}^{\Psi_{0}}\left(F_{n}\right) h_{n} \longmapsto \sum_{n=1}^{N} F_{n} \eta_{n}$ has a unitary extension from $\mathrm{L}_{\mu}^{2}$ to $\int^{\oplus} H_{\mu, \theta}^{\Phi_{0}}\left(\varphi_{0}^{a}\right) \sigma_{\mu, \theta}^{\Phi_{0}}\left(d \varphi_{0}^{a}\right)$ possessing the Property (2.25).

Corollary 3.4. If the representation $\left(V_{\mu, \theta}, \Psi\right)$ has uniform multiplicity, say $n,\left(\right.$ That is, $\operatorname{dim} H_{\mu, \theta}^{\psi}\left(\psi^{a}\right)=n$ for $\sigma_{\mu, \theta}^{\psi}$-a.e. $\left.\psi^{a}\right)$ then we have $\operatorname{dim} H_{\mu, \theta}^{\Phi}\left(\varphi^{a}\right)$ $=n \cdot \operatorname{dim}\left(\mathrm{L}_{Q_{\mu, \theta}}^{t_{J \varphi} a}\right)$ for $\sigma_{\mu, \theta}^{\Phi}-a \cdot e \cdot \varphi^{a}$.

\section{§4. Ergodicity and Multiplicity}

4.1 Ergodicity and uniform multiplicity. As was stated in the Introduction, $U_{\mu}\left(x^{*}\right)$ defined by

$$
U_{\mu}\left(x^{*}\right): h(x) \in \mathrm{L}_{\mu}^{2} \longrightarrow \exp \left(i\left\langle x, x^{*}\right\rangle\right) h(x) \in \mathrm{L}_{\mu}^{2}
$$

satisfies the following commutation relations with $T(F)$.

$$
\begin{gathered}
V_{\mu, \theta}(\varphi) U_{\mu}\left(x^{*}\right)=\exp \left(-i\left\langle\varphi, x^{*}\right\rangle\right) U_{\mu}\left(x^{*}\right) V_{\mu, \theta}(\varphi) \\
T_{\mu, \theta}\left(F_{x^{*}}\right) U_{\mu}\left(x^{*}\right)=U_{\mu}\left(x^{*}\right) T_{\mu, \theta}(F)
\end{gathered}
$$

where $F_{x^{*}}(\cdot)=F\left(\cdot+x^{*}\right)$.

Theorem 4.1. Let $\mu$ be a $\Phi$-quasi-invariant measure on $(X, \mathfrak{B})$. Then the spectral measure $\sigma$ for $\left(V_{\mu, \theta}, \Phi\right)$ is $X^{*}$-quasi-invariant. (More exactly, $X^{*} \mid \Phi$-quasi-invariant.)

Moreover if $\mu$ is $\Phi$-ergodic, (That is, $\mu(B)=1$ or 0 provided that $\mu((B-\varphi) \ominus$ $B)=0$ for all $\varphi \in \Phi)$ then $\sigma$ is also $X^{*}$-ergodic.

Proof. Let $h \in \mathrm{L}_{\mu}^{2}$ and $E \in \bigodot_{\Phi}$. Then

$$
\begin{aligned}
\sigma_{\mu, \theta, h}\left(E-x^{*}\right) & =\left\langle T\left(\left(\chi_{E}\right)_{x^{*}}\right) h, h\right\rangle_{2}=\left\langle T\left(\chi_{E}\right) U_{\mu}\left(-x^{*}\right) h, U_{\mu}\left(-x^{*}\right) h\right\rangle_{2} \\
& =\sigma_{\mu, \theta, U\left(-x^{*}\right) h}(E) .
\end{aligned}
$$

Thus,

$$
\sigma(E)=0 \Longleftrightarrow \forall h, \sigma_{\mu, \theta, h}(E)=0 \Longleftrightarrow \forall h, \sigma_{\mu, \theta, U\left(-x^{*}\right) h}(E)=0 \Longleftrightarrow \sigma\left(E-x^{*}\right)=0 .
$$

If $\sigma\left(\left(E-x^{*}\right) \ominus E\right)=0$ for all $x^{*} \in X^{*}$, then a projection $T\left(\chi_{E}\right)$ commutes with every $U_{\mu}\left(x^{*}\right)$ and $V_{\mu, \theta}(\varphi)$. As the ergodicity of $\mu$ is equivalent to the 
irreducibility of $\left\{U_{\mu}\left(x^{*}\right), V_{\mu, \theta}(\varphi)\right\}$, so $T\left(\chi_{E}\right)=\mathrm{I}$ or 0 and the conclusion follows from the injectivity of $T$.

Theorem 4.2. Put $D_{p, \mu, \theta}:=\left\{\varphi^{a} \in \Phi^{a} \mid \operatorname{dim} H_{\mu, \theta}^{\Phi}\left(\varphi^{a}\right)=p\right\}$. Then

(1) $\sigma_{\mu, \theta}^{\Phi}\left(\left(D_{p, \mu, \theta}-x^{*}\right) \ominus D_{p, \mu, \theta}\right)=0$ for all $x^{*} \in X^{*}$.

(2) There exist at most countable $\Phi$-quasi-invariant measures $\mu_{p}$ such that $\mu_{p}$ are mutually singular with each other, $\mu$ is a convex sum of $\mu_{p}^{\prime} s$ and $\left(V_{\mu_{p}, \theta}, \Phi\right)$ has uniform multiplicity $p$.

(3) In particular, if $\mu$ is $\Phi$-ergodic, then the corresponding representation has a uniform multiplicity.

Proof. Let $h_{1}, h_{2} \in \mathrm{L}_{\mu}^{2}$ and $F \in \mathrm{L}_{\sigma}^{\infty}$. Then

$$
\begin{aligned}
& \int_{\Phi^{a}} F\left(\varphi^{a}\right)\left\langle S_{\mu, \theta} U_{\mu}\left(-x^{*}\right) h_{1} \mid S_{\mu, \theta} U_{\mu}\left(-x^{*}\right) h_{2}\right\rangle\left(\varphi^{a}\right) \sigma\left(d \varphi^{a}\right) \\
& =\int_{\Phi^{a}} F\left(\varphi^{a}+x^{*}\right)\left\langle S_{\mu, \theta} h_{1} \mid S_{\mu, \theta} h_{2}\right\rangle\left(\varphi^{a}\right) \sigma\left(d \varphi^{a}\right) \\
& =\int_{\Phi^{a}} F\left(\varphi^{a}\right)\left\langle S_{\mu, \theta} h_{1} \mid S_{\mu, \theta} h_{2}\right\rangle\left(\varphi^{a}-x^{*}\right) \frac{d \sigma_{x^{*}}}{d \sigma}\left(\varphi^{a}\right) \sigma\left(d \varphi^{a}\right) .
\end{aligned}
$$

So we have

$$
\begin{aligned}
\left\langle S_{\mu, \theta} U_{\mu}\left(-x^{*}\right) h_{1} \mid S_{\mu, \theta} U_{\mu}\left(-x^{*}\right) h_{2}\right\rangle\left(\varphi^{a}\right. & \left.+x^{*}\right) \\
& =\left\langle S_{\mu, \theta} h_{1} \mid S_{\mu, \theta} h_{2}\right\rangle\left(\varphi^{a}\right) \frac{d \sigma_{x^{*}}}{d \sigma}\left(\varphi^{a}+x^{*}\right)
\end{aligned}
$$

for $\sigma$-a.e. $\varphi^{a}$. This leads us the following conclusion that $S_{\mu, \theta} U_{\mu}\left(-x^{*}\right) S_{\mu, \theta}^{-1}$ induces a similar transformation from $H_{\mu, \theta}^{\Phi}\left(\varphi^{a}\right)$ to $H_{\mu, \theta}^{\Phi}\left(\varphi^{a}+x^{*}\right)$ for $\sigma$-a.e. $\varphi^{a}$. Thus $\operatorname{dim} H\left(\varphi^{a}\right)$ is almost all invariant under the actions of $x^{*} \in X^{*}$.

For the second half, note that a projection $T\left(\chi_{D_{p, \mu, \theta}}\right)$ commutes with all $U_{\mu}\left(x^{*}\right)$ and $V_{\mu, \theta}(\varphi)$. Hence there exists $\Omega_{p, \mu, \theta} \in \mathfrak{B}$ such that

$T\left(\chi_{\left.D_{p, \mu, \theta}\right)}\right) h=\chi_{\Omega_{p, \mu, \theta}} \cdot h$ for all $h \in \mathrm{L}_{\mu}^{2}$ and $\mu\left(\left(\Omega_{p, \mu, \theta}-x^{*}\right) \ominus \Omega_{p, \mu, \theta}\right)=0$ for all $\varphi \in \Phi$. Put

$$
\mu_{p, \theta}(B):=\mu\left(\Omega_{p, \mu, \theta}\right)^{-1} \mu\left(B \cap \Omega_{p, \mu, \theta}\right),
$$

for all $B \in \mathfrak{B}$. Then $\mu_{p, \theta}(p=1, \cdots)$ have desired properties.

From Theorem 4.1 and Theorem 4.2 we have a conclusion that the ergodicity of the original measure $\mu$ derives the ergodicity of the spectral measure $\sigma$ and uniform multiplicity $p$. The converse does not hold in general. In fact for the case $p=2, \cdots, \infty$ we have the following example.

Example 2. $X=\boldsymbol{R}^{\infty}, \Phi=\boldsymbol{R}_{0}^{\infty}\left(\boldsymbol{R}_{0}^{\infty}\right.$ is the countable direct sum of $\boldsymbol{R}$.) 


$$
\mu=\sum_{n=1}^{p} \alpha_{n} \nu_{(n-1) \tau} \text { with } \alpha_{n}>0, \sum_{n=1}^{p} \alpha_{n}=1
$$

where $\nu$ is a product type of 1-dimensional measures, which is $\boldsymbol{R}_{0}^{\infty}$-quasiinvariant and $\tau \in \boldsymbol{R}^{\infty}$ is choosen such as $\nu_{n \tau}$ is singular with $\nu$ for every $n$. $\theta$ is a trivial 1-cocycle, so we shall omit the suffix $\theta$. It is obvious that $\mu$ is not $\boldsymbol{R}_{0}^{\infty}$-ergodic for $p \geq 2$. Let $h, g \in \mathrm{L}_{\mu}^{2}$ and $F$ be a bounded measurable function on $\boldsymbol{R}^{\infty}$. Then

$$
\left\langle T_{\mu}(F) h, g\right\rangle_{2}=\sum_{n=1}^{p} \alpha_{n}\left\langle T_{\nu_{(n-1) \tau}}(F) \chi_{X_{n}} \cdot h, \chi_{X_{n}} \cdot g\right\rangle_{2},
$$

where $\left\{X_{n}\right\}_{n}$ is a Borel partition of $\boldsymbol{R}^{\infty}$ such that $\nu_{(n-1) \tau}\left(X_{m}\right)=\delta_{n, m}$. Note that $\sigma_{\nu(n-1) \tau}$ are all equivalent to $\sigma_{\nu}$. So it follows from (4.4) that we have $\sigma_{\mu}=\sigma_{\nu}$, which is $\boldsymbol{R}_{0}^{\infty}$-ergodic, because $\nu$ is $\boldsymbol{R}_{0}^{\infty}$-ergodic.

In order to see the multiplicity, let the expression of the direct integral ( $V_{\nu_{(n-1) \tau}}$, $\left.\boldsymbol{R}_{0}^{\infty}\right)$ be $H^{n}=\int^{\oplus} H^{n}(x) \sigma_{\nu}(d x)$. Then we have $\operatorname{dim} H^{n}(x)=1$ for $\sigma_{\nu}$-a.e. $x$ which will be seen in Section 5. We form a direct sum of Hilbert spaces $H^{n}(x), H(x)$ $:=\sum_{n=1}^{p \oplus} H^{n}(x)$.

Take a sequence $\left\{v_{i}^{n}\right\}_{i} \subset H^{n}$ such that $\left\{v_{i}^{n}(x)\right\}_{i}$ is total in each $H^{n}(x)$. Then $\left\{v_{i_{1}}\right.$, $\cdots, v_{i m}, \cdots(x)=\left(v_{i_{1}}^{1}(x) \cdots, v_{i_{m}}^{m}(x), \cdots\right) \mid v_{i m}^{m}=0$ except finite numbers of $\left.m\right\}$ forms a total sequence at each $H(x)$ and $\left\langle v_{i_{1}}, \cdots, v_{i_{m}}, \cdots \mid v_{j_{1}}, \cdots, v_{j_{m}}, \cdots\right\rangle(x)$ are measurable. Therefore a measurable field structure is induced in such a manner as before and a direct integral $H:=\int^{\oplus} H(x) \sigma_{\nu}(d x)$ is defined. Let us define a map $S$ from $\mathrm{L}_{\mu}^{2}$ to $H$ such that

$$
S(h)=\left(\sqrt{\alpha_{n}} S_{\nu_{(n-1) \tau}}\left(\chi_{X_{n}} \cdot h\right)\right),
$$

where $S_{\nu_{(n-1) \tau}}$ is the canonical map from $\mathrm{L}_{\nu_{(n-1) \tau}}^{2}$ to $H^{n}$. Then

$$
\begin{aligned}
\langle T(F) h, g\rangle_{2} & =\sum_{n=1}^{p} \alpha_{n} \int_{R^{\infty}} F(x)\left\langle S_{\nu(n-1) \tau} \chi_{X_{n}} \cdot h \mid S_{\nu_{(n-1) \tau}} \chi_{X_{n}} \cdot g\right\rangle(x) \sigma_{\nu}(d x) \\
& =\int_{R^{\infty}} F(x)\langle S h \mid S g\rangle(x) \sigma_{\nu}(d x) .
\end{aligned}
$$

Further it is easy to see that $S$ is onto, so $S$ is a unitary map with the desired property. Consequently $\left(V_{\mu}, \boldsymbol{R}_{0}^{\infty}\right)$ has the $\boldsymbol{R}_{0}^{\infty}$-ergodic spectral measure and has uniform multiplicity $p$.

\subsection{Multiplicity 1.}

Proposition 4.1. $\left(V_{\mu, \theta}, \Phi\right)$ is cyclic, if and only if it has uniform multiplicity 1. 
Proof. $\quad(\Leftarrow)$ The sufficiency is obvious.

$(\Rightarrow)$ Let $h_{0}$ be a cyclic element of the representation and put $\eta_{0}:=S_{\mu, \theta} h_{0}$, and $A:=\left\{\varphi^{a} \in \Phi^{a} \mid \eta_{0}\left(\varphi^{a}\right)=0\right\}$. Then

$$
\left\langle T\left(\chi_{A}\right) h, T(F) h_{0}\right\rangle_{2}=\int_{A} \bar{F}\left(\varphi^{a}\right)\left\langle h \mid \eta_{0}\right\rangle\left(\varphi^{a}\right) \sigma_{\mu, \theta}\left(d \varphi^{a}\right)=0
$$

for all $h \in \mathrm{L}_{\mu}^{2}$ and $F \in \mathrm{L}_{\lambda}^{\infty}$, which shows that $\sigma_{\mu, \theta}(A)=0$. New let $\left\{h_{n}\right\}_{n}$ be a sequence from $\mathrm{L}_{\mu}^{2}$ such that $\eta_{n}:=S_{\mu, \theta} h_{n}$ has the properties like (P.6) and (P.7). Set $k_{n}:=S_{\mu, \theta}^{-1}\left(\left\langle\eta_{n} \mid \eta_{0}\right\rangle \eta_{1}-\left\langle\eta_{1} \mid \eta_{0}\right\rangle \eta_{n}\right)$ for $\left.n\right\rangle 1$. Then $\left\langle k_{n}, T(F) \eta_{0}\right\rangle_{2}=0$ and therefore

$$
\left\langle\eta_{0} \mid \eta_{n}\right\rangle\left(\varphi^{a}\right)=0 \text { and }\left\langle\eta_{0} \mid \eta_{1}\right\rangle\left(\varphi^{a}\right) \eta_{n}\left(\varphi^{a}\right)=0
$$

for $\sigma_{\mu, \theta}$-a.e. $\varphi^{a}$. If $\left\langle\eta_{0} \mid \eta_{1}\right\rangle\left(\varphi^{a}\right)=0$ on some set $B \in \mathfrak{C}_{\Phi}$ with positive measure, then it follows from (4.5) that $B \subseteq A$ which contradicts to $\sigma(A)=0$. So $\eta_{n}=0$ for $n$ $\geq 2$.

Theorem 4.3. So far as cyclic representations are concerned,

(1) $\mu$ is $\Phi$-ergodic if and only if $\sigma_{\mu, \theta}$ is $X^{*}$-ergodic.

(2) $\left(V_{\mu, \theta}, \Phi\right) \cong\left(V_{\mu^{\prime}, \theta^{\prime}}, \Phi\right)$, if and only if $\sigma_{\mu, \theta} \cong \sigma_{\mu^{\prime}, \theta^{\prime}}$.

Proof. Suppose that $\sigma_{\mu, \theta}$ is $X^{*}$-ergodic and $\mu((B-\varphi) \ominus B)=0$ for all $\varphi \in$ $\Phi$. We put $P h:=\chi_{B} \cdot h$ for $h \in \mathrm{L}_{\mu}^{2}$, and $Q:=S_{\mu, \theta} P S_{\mu, \theta}^{-1}$. Since $P$ commutes with all $T(F), F \in \mathrm{L}_{\sigma}^{\infty}$, so $Q$ commutes with all $T^{H}(F)$. Thus there exists $E \in \S_{\Phi}$ such that $Q=T^{H}\left(\chi_{E}\right)$. Now

$$
\left\langle T\left(\chi_{E}\right) h, g\right\rangle_{2}=\langle Q S h, S g\rangle_{2}=\langle P h, g\rangle_{2}
$$

for all $h, g \in \mathrm{L}_{\mu}^{2}$, which implies $T\left(\chi_{E}\right)=P$. Besides,

$$
T\left(\left(\chi_{E}\right)_{x^{*}}\right)=U_{\mu}\left(x^{*}\right) T\left(\chi_{E}\right) U_{\mu}\left(-x^{*}\right)=P=T\left(\chi_{E}\right) .
$$

It follows that $\sigma\left(\left(E-x^{*}\right) \ominus E\right)=0$ and therefore $\sigma(E)=1$ or 0 , which is equivalent to $\mu(B)=1$ or 0 . The rest of the proof is immediate.

Of course there is an example of non ergodic measure with cyclic representation.

Example 3. $X=\boldsymbol{R}^{\infty}, \Phi=\boldsymbol{R}_{0}^{\infty}, \quad \mu=\sum_{n=1}^{\infty} \alpha_{n} g_{c_{n}}$, with $\alpha_{n}>0, \quad \sum_{n=1}^{\infty} \alpha_{n}=1$, where $\left\{c_{n}\right\}_{n}$ is a mutually different positive sequence and $g_{c}$ is a standard Gaussian measure with mean 0 and variance $c . \theta$ is a trivial 1 -cocycle, so we shall omit the suffix $\theta$. Later in Section 6, it will be shown that the representation $\left(V_{g_{c}}, \boldsymbol{R}_{0}^{\infty}\right)$ has a spectral measure $g_{(4 c)^{-1}}$ and has uniform multiplicity 1 . For the Gaussian case the spectral measure is attained at constant function. As before, 


$$
\left\langle T_{\mu}(F) h, g\right\rangle_{2}=\sum_{n=1}^{\infty} \alpha_{n}\left\langle T_{g_{c_{n}}}(F) \chi_{X_{n}} F, \chi_{X_{n}} g\right\rangle_{2}
$$

for all $h, g \in \mathrm{L}_{\mu}^{2}$ and $F \in \mathrm{L}_{\sigma_{\mu}}^{\infty}$, where $\left\{X_{n}\right\}_{n}$ is a Borel partion of $\mathbb{R}^{\infty}$ such that $g_{c_{n}}\left(X_{m}\right)=\delta_{n, m}$. It follows that

$$
\sigma_{\mu}=\sum_{n=1}^{\infty} \alpha_{n} \sigma_{g_{c_{n}}}=\sum_{n=1}^{\infty} \alpha_{n} g_{\left(4 c_{n}\right)^{-1}}
$$

so $\sigma_{\mu}$ is not $\mathbb{R}_{0}^{\infty}$-ergodic.

We take another Borel partition $\left\{Y_{n}\right\}_{n}$ such that $g_{\left(4 c_{n}\right)^{-1}}\left(Y_{m}\right)=\delta_{n, m}$. Let $H^{n}=$ $\int_{Y_{n}}^{\oplus} H^{n}(y) \alpha_{n}^{-1} \sigma_{\mu}(d y)$ be a direct integral for $\left(V_{g_{c_{n}}}, \boldsymbol{R}_{0}^{\infty}\right)$ and $S_{n}$ be the canonical map from $\mathrm{L}_{g_{c_{n}}}^{2}$ to $H^{n}$, and put $H(y):=H^{n}(y)$, if $y \in Y_{n}$. As for the measurable field structure on $H(y)$ 's, we shall consider induced one from each measurable field structure on $H(y)$ 's. Now we shall show that $\mathrm{L}_{\mu}^{2}$ is isomorphic to a direct integral $H:=\int_{R^{\infty}}^{\oplus} H(y) \sigma_{\mu}(d y)$ by a map $S$ defined by $(S h)(y)=S_{n}\left(\chi_{x_{n}} h\right)(y)$ for $y \in Y_{n}$. For,

$$
\begin{aligned}
\left\langle T_{\mu}(F) h, g\right\rangle_{2} & =\sum_{n=1}^{\infty} \alpha_{n} \int_{Y_{n}} F(y)\left\langle S_{n}\left(\chi_{x_{n}} h\right) \mid S_{n}\left(\chi_{x n} g\right)\right\rangle(y) \alpha_{n}^{-1} \sigma_{\mu}(d y) \\
& =\int_{R^{\infty}} F(y)\langle S h \mid S g\rangle(y) \sigma_{\mu}(d y) .
\end{aligned}
$$

Thus $\left(V_{\mu}, \boldsymbol{R}_{0}^{\infty}\right)$ is cyclic, however $\mu$ is not $\boldsymbol{R}_{0}^{\infty}$-ergodic.

4.3 Unitary cocycles. We have seen that the ergodicity of the spectral measure and the uniform multiplicity 1 implies that the original measure is ergodic and that the ergodicity of the original measure implies that the ergodicity of the spectral measure and a uniform multiplicity.

However we don't yet know whether the uniform multiplicity can be taken the place of uniform multiplicity 1 .

Let us make the following device in order to approach to this problem.

Let $\mu$ be $\Phi$-quasi-invariant, $\left(V_{\mu, \theta}, \Phi\right)$ have uniform multiplicity $p$, and $K$ be a Hilbert space of dimension $p$. Then $\mathrm{L}_{\mu}^{2}$ is canonically isomorphic to $\mathrm{L}_{\sigma}^{2}(K)$ of all square summable $K$-valued functions by a map $S_{\mu, \theta}$. Put

$$
\widetilde{V_{\mu, \theta}}(\varphi)=S_{\mu, \theta} V_{\mu, \theta}(\varphi) S_{\mu, \theta}^{-1} \text { and } \widetilde{U_{\mu, \theta}}\left(\varphi^{*}\right)=S_{\mu, \theta} U_{\mu}\left(\varphi^{*}\right) S_{\mu, \theta}^{-1}
$$

for all $\varphi \in \Phi$ and $\varphi^{*} \in X^{*}$. Then it follows from (4.1) that

$$
\left(\widetilde{U_{\mu, \theta}}\left(\varphi^{*}\right) k\right)\left(\varphi^{a}\right)=\exp \left(-i\left\langle\varphi, \varphi^{a}+\varphi^{*}\right\rangle\right) \widetilde{U_{\mu, \theta}}\left(\varphi^{*}\right)(\exp (i\langle\varphi, \cdot\rangle) k)\left(\varphi^{a}\right)
$$

for all $k \in K$ and

$$
\int_{\Phi^{a}} \exp \left(i\left\langle\varphi, \varphi^{a}\right\rangle\right)\left\|\widetilde{U_{\mu, \theta}}\left(\varphi^{*}\right) k\right\|_{K}^{2} \sigma\left(d \varphi^{a}\right)
$$




$$
=\|k\|_{K}^{2} \exp \left(-i\left\langle\varphi, \varphi^{*}\right\rangle\right) \int_{\Phi^{a}} \exp \left(i\left\langle\varphi, \varphi^{a}\right\rangle\right) \sigma\left(d \varphi^{a}\right) .
$$

Thus, we have

$$
\left\|\widetilde{U_{\mu, \theta}}\left(\varphi^{*}\right) k\right\|_{K}=\sqrt{\frac{d \sigma_{-\varphi *}}{d \sigma}}\left(\varphi^{a}\right)\|k\|_{K}
$$

for $\sigma$-a.e. $\varphi^{a}$. Therefore $U_{\mu, \theta}\left(\varphi^{a}, \varphi^{*}\right)$ defined by

$$
U_{\mu, \theta}\left(\varphi^{a}, \varphi^{*}\right) k=\left(\frac{d \sigma_{-\varphi^{*}}}{d \sigma}\left(\varphi^{a}\right)\right)^{-1 / 2}\left(\widetilde{U_{\mu, \theta}}\left(\varphi^{*}\right) k\right)\left(\varphi^{a}\right)
$$

is unitary for $\sigma$-a.e. $\varphi^{a}$. Consequently,

$$
\left(\widetilde{U_{\mu, \theta}}\left(\varphi^{*}\right) f\right)\left(\varphi^{a}\right)=\sqrt{\frac{d \sigma_{-\varphi^{*}}}{d \sigma}}\left(\varphi^{a}\right) U_{\mu, \theta}\left(\varphi^{a}, \varphi^{*}\right) f\left(\varphi^{a}+\varphi^{*}\right)
$$

for all $f \in \mathrm{L}_{\sigma}^{2}(K)$. It is easily checked that

$$
\begin{gathered}
U_{\mu, \theta}\left(\varphi^{a}, \varphi_{2}^{*}\right) U_{\mu, \theta}\left(\varphi^{a}+\varphi_{2}^{*}, \varphi_{1}^{*}\right)=U_{\mu, \theta}\left(\varphi^{a}, \varphi_{1}^{*}+\varphi_{2}^{*}\right) \\
U_{\mu, \theta}\left(\varphi^{a}, 0\right)=\mathrm{I}
\end{gathered}
$$

for $\sigma$-a.e. $\varphi^{a}$. We call a system of unitary operators possessing the properties (4.12) and (4.13) a unitary cocycle.

Conversely, suppose that a $\Phi^{*}$-quasi-invariant probability measure $\sigma$ on $\left(\Phi^{a}\right.$, $\left.\mathfrak{\Im}_{\Phi}\right)$, complex separable Hilbert space $K$ and a unitary cocycle $\left\{U\left(\varphi^{a}, \varphi^{*}\right)\right\}$ are given. We define unitary operators $\widetilde{V}(\varphi), \widetilde{U}\left(\varphi^{*}\right)$ on $\mathrm{L}_{\sigma}^{2}(K)$ such that

$$
\begin{aligned}
\widetilde{V}(\varphi) & : f\left(\varphi^{a}\right) \longmapsto \exp \left(i\left\langle\varphi, \varphi^{a}\right\rangle\right) f\left(\varphi^{a}\right) \\
\widetilde{U}\left(\varphi^{*}\right) & : f\left(\varphi^{a}\right) \longmapsto \sqrt{\frac{d \sigma_{-\varphi^{*}}}{d \sigma}}\left(\varphi^{a}\right) U\left(\varphi^{a}, \varphi^{*}\right) f\left(\varphi^{a}+\varphi^{*}\right) .
\end{aligned}
$$

If $\widetilde{U}\left(\varphi^{*}\right)$ is a cyclic representation of $\Phi^{*}$ and the representations $\left\{\tilde{V}(\varphi), \widetilde{U}\left(\varphi^{*}\right)\right\}$ is irreducible, then it follows that there correspondes a $\Phi$-ergodic measure $\mu$ on $\left(\Phi^{*}\right)^{a}$ such that

$$
\left\langle\widetilde{U}\left(\varphi^{*}\right) h_{0}, h_{0}\right\rangle_{2}=\int_{\left(\Phi^{*}\right) a} \exp \left(i\left\langle\varphi^{*}, x\right\rangle\right) \mu(d x),
$$

where $h_{0} \in \mathrm{L}_{\sigma}^{2}(K)$ is a cyclic vector with $\left\|h_{0}\right\|_{2}=1$. So an operator $S^{-1}$ defined by

$$
S^{-1}: \sum_{n=1}^{N} \alpha_{n} \tilde{U}\left(\varphi_{n}^{*}\right) h_{0} \in \mathrm{L}_{\sigma}^{2}(K) \longmapsto \sum_{n=1}^{N} \alpha_{n} \exp \left(i\left\langle\varphi_{n}^{*}, \cdot\right\rangle\right) \in \mathrm{L}_{\mu}^{2}
$$

has a unitary extension denoted by the same letter, and $U\left(\varphi^{*}\right):=S^{-1} \widetilde{U}\left(\varphi^{*}\right) S$ and $V(\varphi):=S^{-1} \widetilde{V}(\varphi) S$ have the following explicit form. 


$$
\begin{aligned}
& U\left(\varphi^{*}\right): h(x) \longmapsto \exp \left(i\left\langle\varphi^{*}, x\right\rangle\right) h(x) \\
& V(\varphi): h(x) \longmapsto \sqrt{\frac{d \mu_{\varphi}}{d \mu}}(x) \theta(x, \varphi) h(x-\varphi),
\end{aligned}
$$

where $\theta$ is some 1-cocycle. (For these discussions, see Chapter IV in [3].) Therefore an original situation is realized as $X=\left(\Phi^{*}\right)^{a}$ with weak topology. Consequently in order to give a counter example for this problem it is enough to construct a measure $\sigma$ and a unitary cocycle $\left\{U\left(\varphi^{a}, \varphi^{*}\right)\right\}$ on $\mathrm{L}_{\sigma}^{2}(K)$ with $\operatorname{dim}(K) \geq 2$ such that $\widetilde{U}\left(\varphi^{*}\right)$ is cyclic and $\left\{\widetilde{U}\left(\varphi^{*}\right), \widetilde{V}(\varphi)\right\}$ is irreducible.

A typical example of a unitary cocycle is given by

$$
U\left(\varphi^{a}, \varphi^{*}\right)=U\left(\varphi^{a}\right)^{-1} U\left(\varphi^{a}+\varphi^{*}\right),
$$

where $U$ is a measureable map from $\Phi^{a}$ to the unitary group on $K$ equipped with a Borel field generated by the strong operator topology. However the representation $\left\{\widetilde{U}\left(\varphi^{*}\right), \widetilde{V}(\varphi)\right\}$ derived from this cocycle is equivalent to a representation $\left\{\widetilde{U}_{0}\left(\varphi^{*}\right), \widetilde{V}_{0}(\varphi)\right\}$ derived from the trivial unitary cocycle by a map, $f\left(\varphi^{a}\right) \longmapsto U\left(\varphi^{a}\right)^{-1} f\left(\varphi^{a}\right)$. So it derives a non irreducible representation, if $\operatorname{dim}(K) \geq 2$. Similarly if $\left\{U\left(\varphi^{a}, \varphi^{*}\right)\right\}$ consists of commutative operators, then the corresponding representation is reducible.

We finally remark that in the case $X=\boldsymbol{R}^{\infty}, \Phi=\boldsymbol{R}_{0}^{\infty}$, an explicit form of unitary cocycle is decided as follows.

$$
U(x, s)=U_{1}\left(x^{1}\right) \cdots U_{n}\left(x^{n}\right) U_{n}\left((x+s)^{n}\right)^{-1} \cdots U_{1}\left((x+s)^{1}\right)^{-1},
$$

where $x=\left(x_{1}, \cdots, x_{n}, \cdots\right), x^{n}=\left(x_{n}, x_{n+1}, \cdots\right) \in \boldsymbol{R}^{\infty}, s=\left(s_{1}, \cdots, s_{n}, 0,0, \cdots\right) \in \boldsymbol{R}_{0}^{\infty}$ and $U_{n}$ is a measurable map from $\boldsymbol{R}^{\infty}$ to the unitary group on $K$.

Anyway, it seems to the author that this problem will be solved negatively. And if so, it is quite interesting to construct a multiplicity formula for ergodic measures.

\section{§5. Product Representation}

5.1 Finite product. Let $X_{n}(n=1, \cdots, N<\infty)$ be a locally convex Hausdorff space over $\mathbb{R}, \mathfrak{B}_{n}$ be the cylindrical $\sigma$-algebra on $X_{n}, \mu_{n}$ be a $\Phi_{n}$-quasi-invariant probability measure on $\left(X_{n}, \mathfrak{B}_{n}\right)$ and $\theta_{n}$ be a 1-cocycle with property (2.4). Put

$$
X:=X_{1} \times \cdots \times X_{N}, \mathfrak{B}:=\mathfrak{B}_{1} \times \cdots \times \mathfrak{B}_{N} \text { and } \mu:=\mu_{1} \times \cdots \times \mu_{N} .
$$

It is easily checked that $\mathfrak{B}$ coincides with the cylindrical $\sigma$-algebra on $X$ and 
that $A_{\mu}^{0} \supseteq \Phi:=\Phi_{1} \times \cdots \times \Phi_{N}$. So a unitary representation of $\Phi$ is defined as

$$
\begin{aligned}
& V_{\mu, \theta}\left(\left(\varphi_{1}, \cdots, \varphi_{N}\right)\right): f\left(x_{1}, \cdots, x_{N}\right) \in \mathrm{L}_{\mu}^{2} \longmapsto \\
& \prod_{n=1}^{N} \sqrt{\frac{d\left(\mu_{n}\right)_{\varphi_{n}}}{d \mu_{n}}}\left(x_{n}\right) \theta_{n}\left(x_{n}, \varphi_{n}\right) f\left(x_{1}-\varphi_{1}, \cdots, x_{N}-\varphi_{N}\right) \in \mathrm{L}_{\mu}^{2} .
\end{aligned}
$$

In this subsection first we shall find an expression of the direct integral for $\left(V_{\mu, \theta}\right.$, $\Phi)$ using factor expressions.

So, choose $h_{n}^{0} \in \mathrm{L}_{\mu_{n}}^{2}$ such that $\sigma_{\mu_{n}, \theta_{n}, h_{n}}=\sigma_{\mu_{n}, \theta_{n}}\left(=: \sigma_{n}\right)$ for each $n$, and set $\sigma:=\sigma_{1}$ $\times \cdots \times \sigma_{N}$. Since we have

$$
\begin{aligned}
& \left\langle T_{\mu, \theta}\left(F_{1} \otimes \cdots \otimes F_{N}\right)\left(h_{1} \otimes \cdots \otimes h_{N}\right), g_{1} \otimes \cdots \otimes g_{N}\right\rangle_{2} \\
& \quad=\prod_{n=1}^{N}\left\langle T_{\mu_{n}}\left(F_{n}\right) h_{n}, g_{n}\right\rangle_{2}=\int_{\Phi^{a}} \prod_{n=1}^{N} F_{n}\left(\varphi_{n}^{a}\right)\left\langle S_{\mu_{n}, \theta_{n}}^{\Phi_{n}} h_{n} \mid S_{\mu_{n, \theta}}^{\Phi_{n}} g_{n}\right\rangle\left(\varphi_{n}^{a}\right) \sigma\left(d \varphi^{a}\right)
\end{aligned}
$$

for all $h_{n}, g_{n} \in \mathrm{L}_{\mu_{n}}^{2}$ and for all $F_{n} \in \mathrm{L}_{\sigma_{n}}^{\infty}$, it follows that $\sigma_{\mu, \theta}=\sigma$, and it is natural to define

$$
H_{\mu, \theta}\left(\varphi^{a}\right)=\bigotimes_{n=1}^{N} H_{\mu_{n}, \theta_{n}}\left(\varphi_{n}^{a}\right)
$$

for $\varphi^{a}=\left(\varphi_{1}^{a}, \cdots, \varphi_{N}^{a}\right)$. Next a measurable field structure $R$ is defined such that $\eta\left(\varphi^{a}\right) \in R$ if and only if $\left\langle\eta\left(\varphi^{a}\right) \mid \eta_{1}\left(\varphi_{1}^{a}\right) \otimes \cdots \otimes \eta_{N}\left(\varphi_{N}^{a}\right)\right\rangle$ is measurable for each $\eta_{n}$ $\in R^{\Phi_{n}}(n=1, \cdots, N)$. Thus a direct integral $H=\int^{\oplus} H_{\mu, \theta}\left(\varphi^{a}\right) \sigma\left(d \varphi^{a}\right)$ is constructed and it is easily checked that a map, $h_{1} \otimes \cdots \otimes h_{N} \longmapsto \bigotimes_{n=1}^{N}\left(S_{\mu_{n}, \theta_{n}} h_{n}\right)\left(\varphi_{n}^{a}\right)$ has a unitary extension from $\mathrm{L}_{\mu}^{2}$ to $H$. Settling these arguments,

Theorem 5.1. An expression of the direct integral for $\left(V_{\mu, \theta}, \Phi\right)$ is $\int^{\oplus} \otimes_{n=1}^{N} H_{\mu_{n, \theta}}\left(\varphi_{n}^{a}\right) \sigma\left(d \varphi^{a}\right)$. Thus the spectral measure $\sigma$ is the product of each spectral measure of the factor and the multiplicity is the product of each multiplicity.

Here we shall make addition to the ergodicity of $\mu$ for a little while.

Theorem 5.2. Under the same notation as in this paragraph, if $\mu_{n}$ is $\Phi_{n}$-ergodic for each $n$, then $\mu$ is $\Phi$-ergodic.

Proof. It is enough to show it in the case $N=2$. The general case follows from the mathematical induction.

So let $A \in \mathfrak{B}$ and $\mu\left(\left(A-\left(\varphi_{1}, \varphi_{2}\right)\right) \ominus A\right)=0$ for all $\varphi_{j} \in \Phi_{j}(j=1,2)$. Then there exists a countable set $\left\{x_{j, m}^{*}\right\}_{m} \subset X_{j}^{*}(j=1,2)$ such that $A \in \mathfrak{C}_{1} \times \mathfrak{\mho}_{2}$, where $\mathfrak{\mho}_{j}$ is the minimal $\sigma$-algebra with which all the $x_{j, m}^{*}(m=1, \cdots)$ are measurable.

Now let us define a metric on $\Phi_{j}$ such that 


$$
d^{j}(\varphi, \psi):=\left\|\left(\widehat{\mu_{j}}\right)_{\varphi}-\left(\widehat{\mu_{j}}\right)_{\psi}\right\|_{\text {tot }},
$$

where $\widehat{\mu_{j}}=\mu_{j} \mid \S_{j}$. Since $\mathrm{L}_{\widehat{\mu_{j}}}^{2}$ is separable, so is $\left(\Phi_{j}, d^{j}\right)$. We take a countable dense set $\left\{\varphi_{j, m}\right\}_{m}$ from $\left(\Phi_{j}, d^{j}\right)$. Then using Fubini's theorem, we see that there exists some $N_{1} \in \mathfrak{夭}_{2}$ such that $\mu_{2}\left(N_{1}\right)=0$ and for all $y \in N_{1}^{c}$

$$
\mu_{1}\left(\left(A^{y}-\varphi_{1, m}\right) \ominus A^{y}\right)=0
$$

for all $m$. Since $d^{1}\left(\psi_{n}, \psi\right) \longrightarrow 0$ implies $\mu_{1}\left(\left(E-\psi_{n}\right) \ominus(E-\psi)\right) \longrightarrow 0$ for any $E$ $\in \mathfrak{C}_{1}$, which will be shown later soon, so for all $y \in N_{1}^{c}$

$$
\mu_{1}\left(\left(A^{y}-\varphi\right) \ominus A^{y}\right)=0
$$

for all $\varphi \in \Phi_{1}$. It follows from the ergodicity of $\mu_{1}$ that

$$
\mu_{1}\left(A^{y}\right)=0 \text { or } 1 \text {. }
$$

Notice that $x \in\{A-(0, \varphi)\}^{y} \Longleftrightarrow(x, y+\varphi) \in A \Longleftrightarrow x \in A^{y+\varphi}$. Hence for any $\varphi \in \Phi_{2}$

$$
\mu_{1}\left(A^{y+\varphi} \ominus A^{y}\right)=0
$$

for $\mu_{2}$-a.e. $y$ in virtue of Fubini's theorem. Thus we have

$$
\mu_{1}\left(A^{y \pm \varphi_{2, m}} \ominus A^{y}\right)=0(m=1, \cdots)
$$

for all $y \in N_{2}^{c}$, where $N_{2}^{c}$ is some $\mu_{2}$-negligible set. Now put $F:=\left\{y \in \Phi_{2} \mid \mu_{1}\left(A^{y}\right)\right.$ $=1$. It follows from (5.7) that $F \cap N_{2}^{c} \subseteq\left(F \pm \varphi_{2, m}\right) \cap N_{2}^{c}$. Hence discussing in the same way as above, we see that $\mu_{2}(F)=1$ or 0 . Consequently,

$$
\mu(A)=\int_{X_{2}} \mu_{1}\left(A^{y}\right) \mu_{2}(d y)=\int_{F \cap N_{1}^{c}} \mu_{1}\left(A^{y}\right) \mu_{2}(d y)=\mu_{2}(F)=1 \text { or } 0 .
$$

Lemma 5.1. If $d^{1}\left(\psi_{n}, 0\right) \longrightarrow 0(n \longrightarrow \infty)$, then $\widehat{\mu_{1}}\left(\left(E-\psi_{n}\right) \ominus E\right) \longrightarrow 0$ $(n \longrightarrow \infty)$ for each fixed $E \in \mathbb{C}_{1}$.

Proof. It is clear that for any $\epsilon>0$, there exists a function $F(x)$ of the form, $F(x)=f\left(\left\langle x, x_{1,1}^{*}\right\rangle, \cdots,\left\langle x, x_{1, k}^{*}\right\rangle\right)$, where $f$ is a continuous bounded function on $\boldsymbol{R}^{k}$ with $\|f\|_{\infty}=1$ such that $\left\|F-\chi_{E}\right\|_{1}<\epsilon$. It follows that

$$
\begin{aligned}
\widehat{\mu_{1}}\left(\left(E-\psi_{n}\right) \ominus E\right) & =\int_{X_{1}}\left|\chi_{E}\left(x+\psi_{n}\right)-\chi_{E}(x)\right| \widehat{\mu_{1}}(d x) \\
\leq & \int_{X_{1}}\left|\chi_{E}\left(x+\psi_{n}\right)-F\left(x+\psi_{n}\right)\right| \widehat{\mu_{1}}(d x)+\int_{X_{1}} \mid F\left(x+\psi_{n}\right) \\
& -F(x)\left|\widehat{\mu_{1}}(d x)+\int_{X_{1}}\right| F(x)-\chi_{E}(x) \mid \widehat{\mu_{1}}(d x) \\
<2 \epsilon & +\int_{X_{1}}\left|F\left(x+\psi_{n}\right)-F(x)\right| \widehat{\mu_{1}}(d x)+2 \int_{X_{1}}\left|\frac{d\left(\widehat{\mu_{1}}\right)_{\psi_{n}}}{d \widehat{\mu_{1}}}(x)-1\right| \widehat{\mu_{1}}(d x) .
\end{aligned}
$$


The last term in the right hand of the last inequality converges to 0 due to the definition of $d^{1}$, so we only have to check that $\left\langle\psi_{n}, x^{*}\right\rangle \longrightarrow 0(n \longrightarrow \infty)$ for any $x^{*} \in\left\{x_{1,1}^{*}, \cdots, x_{1, k}^{*}\right\}$. Now let $B \in g^{2}(\boldsymbol{R})$ and put $p=x^{*} \mu_{1}$. Then

$$
p\left(B-\left\langle\psi_{n}, x^{*}\right\rangle\right)=\mu_{1}\left(\left(x^{*}\right)^{-1}(B)-\psi_{n}\right) \longrightarrow p(B)(n \longrightarrow \infty) .
$$

Thus $\left\langle\psi_{n}, x^{*}\right\rangle \longrightarrow 0(n \longrightarrow \infty)$, as is easily seen.

5.2 Countably infinite product. As before, let $X_{n}$ be a locally convex Hausdorff space over $\boldsymbol{R}, \mathfrak{B}_{n}$ be the cylindrical $\sigma$-algebra on $X_{n}, \mu_{n}$ be a probability measure on $\left(X_{n}, \mathfrak{B}_{n}\right)$ such that $\Phi_{n} \subset A_{\mu_{n}}^{\circ}$ and $\theta_{n}$ be a 1-cocycle. We put $X:=\prod_{n=1}^{\infty} X_{n}, \mathfrak{B}:=\prod_{n=1}^{\infty} \mathfrak{B}_{n}$ and $\mu:=\prod_{n=1}^{\infty} \mu_{n}$. It is easily checked that $\mathfrak{B}$ coincides with the cylindrical $\sigma$-algebra on $X$, and $A_{\mu}^{\circ} \supset \Phi:=\left\{\left(\varphi_{n}\right) \in \prod_{n=1}^{\infty} \Phi_{n} \mid \varphi_{n}\right.$ $=0$ except finite numbers of $n\}$. Further if $\Phi_{n}=A_{\mu_{n}}^{\circ}$ holds for all $n$, then $\Phi$ is dense in $A_{\mu}^{0}$ with respect to the Kakutani's metric $d_{\mu}$. We put

$$
\theta(x, \varphi):=\prod_{n=1}^{\infty} \theta_{n}\left(x_{n}, \varphi_{n}\right)
$$

for $x=\left(x_{1}, \cdots, x_{n}, \cdots\right) \in X$ and $\varphi=\left(\varphi_{1}, \cdots, \varphi_{n}, \cdots\right) \in \Phi$.

In this subsection, we shall find a direct integral expression for the representation $\left(V_{\mu, \theta}, \Phi\right)$. So let $\mathrm{L}_{\mu_{n}}^{2}$ be canonically isomorphic to $H_{n}:=\int^{\oplus} H_{\mu_{n}, \theta_{n}}$ $\left(\varphi_{n}^{a}\right) \sigma_{n}\left(d \varphi_{n}^{a}\right)$ by a map $S_{n}$. We shall write $\hat{1}_{n}$ in place of $S_{n}(1)$. First we shall take a positive measurable function $\rho_{n}\left(\varphi_{n}^{a}\right)$ such that

$$
\begin{gathered}
\int_{\Phi_{n}^{a}} \rho\left(\varphi_{n}^{a}\right) \sigma_{n}\left(d \varphi_{n}^{a}\right)=1 \text { and } \\
\sum_{n=1}^{\infty}\left\{1-\int_{\Phi_{n}^{a}} \sqrt{\left\langle\hat{1}_{n} \mid \hat{1}_{n}\right\rangle\left(\varphi_{n}^{a}\right)} \sqrt{\rho_{n}\left(\varphi_{n}^{a}\right)} \sigma_{n}\left(d \varphi_{n}^{a}\right)\right\}^{1 / 2}<\infty .
\end{gathered}
$$

Such $\rho_{n}$ surely exists. For example it may be as well to take $C_{\epsilon}^{-1}\left(\left\langle\hat{1}_{n} \mid \hat{1}_{n}\right\rangle\left(\varphi_{n}^{a}\right)\right.$ $+\epsilon$ ) for sufficiently small $\epsilon$, where $C_{\epsilon}$ is the normalizing constant.

Next we change each spectral measure $\sigma_{n}\left(d \varphi_{n}^{a}\right)$ to $\sigma_{n}^{0}\left(d \varphi_{n}^{a}\right):=\rho_{n}\left(\varphi_{n}^{a}\right) \sigma_{n}\left(d \varphi_{n}^{a}\right)$, so the inner product of $H_{\mu_{n}, \theta_{n}}\left(\varphi_{n}^{a}\right)$ is altered from $\langle\cdot \mid \cdot\rangle$ to $\rho_{n}^{-1 / 2}\langle\cdot \mid \cdot\rangle$ which will be denoted by $\langle\cdot \mid \cdot\rangle_{\text {o }}$. Consequently we can rewrite (5.10) in a new form as

$$
\sum_{n=1}^{\infty}\left\{1-\int_{\Phi_{n}^{a}} \sqrt{\left\langle\hat{1}_{n} \mid \hat{1}_{n}\right\rangle \cdot\left(\varphi_{n}^{a}\right)} \sigma_{n}^{\circ}\left(d \varphi_{n}^{a}\right)\right\}^{1 / 2}<\infty .
$$

It follows from (5.11) that $\left\{\prod_{n=1}^{N} \sqrt{\left\langle\hat{1}_{n} \mid \hat{1}_{n}\right\rangle_{\circ}}\left(\varphi_{n}^{a}\right)\right\}_{N}$ forms a Cauchy sequence in $\mathrm{L}_{\sigma}^{2}$, where $\sigma:=\prod_{n=1}^{\infty} \sigma_{n}^{\circ}$ is a probability measure on $\Phi^{a}$. Consequently we have

$$
\int_{\Phi a}\left|\prod_{n=N+1}^{\infty}\left\langle\hat{1}_{n} \mid \hat{1}_{n}\right\rangle_{\circ}\left(\varphi_{n}^{a}\right)-1\right| \sigma\left(d \varphi^{a}\right) \longrightarrow 0(N \longrightarrow \infty) .
$$

Now let $h, g \in \mathrm{L}_{\mu}^{2}$ be tame functions of the form of separation variables, $h=h_{1}$ 
$\otimes \cdots \otimes h_{N}$ and $g=g_{1} \otimes \cdots \otimes g_{N}$, where $h_{n}, g_{n} \in \mathrm{L}_{\mu_{n}}^{2}$ for all $n=1, \cdots, N$. Since we have for $N \leq M$,

$$
\begin{aligned}
& \left\langle V_{\mu, \theta}\left(\left(\varphi_{1}, \cdots, \varphi_{n}, 0,0, \cdots\right)\right) h, g\right\rangle_{2} \\
& =\prod_{n=1}^{N} \int_{\Phi_{n}^{a}} \exp \left(i\left\langle\varphi_{n}, \varphi^{a}\right\rangle\right)\left\langle S_{n} h_{n} \mid S_{n} g_{n}\right\rangle\left(\varphi_{n}^{a}\right) \sigma_{n}^{\circ}\left(d \varphi_{n}^{a}\right) \cdot \\
& \prod_{N+1}^{M} \int_{\Phi_{n}^{a}}^{n} \exp \left(i\left\langle\varphi_{n}, \varphi^{a}\right\rangle\right)\left\langle\hat{1}_{n} \mid \hat{1}_{n}\right\rangle_{\circ}\left(\varphi_{n}^{a}\right) \sigma_{n}^{\circ}\left(d \varphi_{n}^{a}\right),
\end{aligned}
$$

so we have for each tame function $F \in \mathrm{L}_{\sigma}^{\infty}$,

(5.13) $\left\langle T_{\mu, \theta}(F) h, g\right\rangle_{2}$

$$
=\int_{\Phi^{a}} F\left(\varphi^{a}\right) \prod_{n=1}^{N}\left\langle S_{n} h_{n} \mid S_{n} g_{n}\right\rangle\left(\varphi_{n}^{a}\right) \prod_{n=N+1}^{\infty}\left\langle\hat{1}_{n} \mid \hat{1}_{n}\right\rangle_{0}\left(\varphi_{n}^{a}\right) \sigma\left(d \varphi^{a}\right) .
$$

It follows from Theorem 2.1 that we have $\sigma_{\mu, \theta}$ is absolutely continuous with $\sigma$. On the other hand, $\sigma_{\mu, \theta}(E)=0$ gives that $\int_{E} \prod_{n=N+1}^{\infty}\left\langle\hat{1}_{n} \mid \hat{1}_{n}\right\rangle_{0}\left(\varphi_{n}^{a}\right) \sigma\left(d \varphi^{a}\right)=0$ for all $n$, because we only have to substitute the corresponding one to $\eta_{1}$ in (P.6) and (P.7) for $h_{n}$ and $g_{n}$. Hence $\sigma(E)=0$ by virtue of (5.12), and $\sigma$ is regarded as the spectral measure. Here we shall construct a Hilbert space $H\left(\varphi^{a}\right)$ for each $\varphi^{a}=\left(\varphi_{n}^{a}\right) \in \prod_{n=1}^{\infty} \Phi_{n}^{a}=\Phi^{a}$ as follows.

First we notice that (5.11) is nothing else but that $\left\{\hat{1}_{n}\left(\varphi_{n}^{a}\right)\right\}_{n} \in \prod_{n=1}^{\infty} H_{\mu_{n}, \theta_{n}}\left(\varphi_{n}^{a}\right)$ forms a $C_{0}$-sequence for $\sigma$-a.e. $\varphi^{a}$. (For $C_{0}$-sequence, we refer p21 in [6].) Let us put $H_{\mu, \theta}\left(\varphi^{a}\right):=\bigotimes_{n=1}^{\infty(\hat{1})} H_{\mu_{n}, \theta_{n}}\left(\varphi_{n}^{a}\right)$, which is the $(\hat{1})$-adic incomplete direct product. A measurable field structure $R$ is induced in such a manner as $\eta\left(\varphi^{a}\right)$ $\in R$ if and only if $\left\langle\eta\left(\varphi^{a}\right) \mid \eta_{1}\left(\varphi_{1}^{a}\right) \otimes \cdots \otimes \eta_{n}\left(\varphi_{n}^{a}\right) \otimes \cdots\right\rangle$ are measurable for all $\eta_{n} \in$ $R^{\Phi_{n}}$, where $\eta_{n}\left(\varphi_{n}^{a}\right)=\hat{1}_{n}\left(\varphi_{n}^{a}\right)$ except finite numbers of $n$.

Theorem 5.3. $\mathrm{L}_{\mu}^{2}$ is isomorphic to $H:=\int^{\oplus} H_{\mu, \theta}\left(\varphi^{a}\right) \sigma\left(d \varphi^{a}\right)$ with a map $S$ possesing the property (2.25).

Proof. Put for a tame function $h=h_{1} \otimes \cdots \otimes h_{N} \in \mathrm{L}_{\mu}^{2}$,

$$
S h:=\left(S_{\mu_{1}, \theta_{1}} h_{1}\right)\left(\varphi_{1}^{a}\right) \otimes \cdots \otimes\left(S_{\mu_{N}, \theta_{N}} h_{N}\right)\left(\varphi_{N}^{a}\right) \otimes \hat{1}_{N+1}\left(\varphi_{N+1}^{a}\right) \otimes \cdots .
$$

Then (5.13) is rewritten as

$$
\left\langle T_{\mu, \theta}(F) h, g\right\rangle_{2}=\int_{\Phi^{a}} F\left(\varphi^{a}\right)\langle S h \mid S g\rangle\left(\varphi^{a}\right) \sigma\left(d \varphi^{a}\right)
$$

for all $F \in \mathrm{L}_{\sigma}^{\infty}$ and for all tame functions $h, g \in \mathrm{L}_{\mu}^{2}$. The rest of the proof is easily checked.

Corollary 5.4. If all the $\left(V_{\mu_{n}, \theta_{n}}, \Phi_{n}\right)$ are cyclic representations, then so is their product representation $\left(V_{\mu, \theta}, \Phi\right)$. 
Theorem 5.5. Assume that $\mu_{n}^{\prime}(n=1, \cdots)$ be another $\Phi_{n}$-quasi-invariant probability measure on $\left(X_{n}, \mathfrak{B}_{n}\right), \theta_{n}^{\prime}$ be a 1-cocycle, and the representations $\left(V_{\mu_{n}, \theta_{n}}, \Phi_{n}\right)$ and $\left(V_{\mu_{n}^{\prime}, \theta_{n}^{\prime}}, \Phi_{n}\right)$ be equivalent for all $n$. Put $\mu^{\prime}:=\prod_{n=1}^{\infty} \mu_{n}^{\prime}$ and $\theta^{\prime}$ $:=\prod_{n=1}^{\infty} \theta_{n}^{\prime}$. Then in order that $\left(V_{\mu, \theta}, \Phi\right) \cong\left(V_{\mu^{\prime}, \theta^{\prime}}, \Phi\right)$, it is necessary and sufficient that the spectral measures $\sigma_{\mu, \theta}$ and $\sigma_{\mu^{\prime}, \theta^{\prime}}$ are equivalent. And this condition is equivalent to

$$
\sum_{n=1}^{\infty} d^{2}\left\{\left\langle\hat{1}_{n} \mid \hat{1}_{n}\right\rangle\left(\varphi_{n}^{a}\right) \sigma_{\mu_{n}, \theta_{n}}\left(d \varphi_{n}^{a}\right),\left\langle\hat{1}_{n}^{\prime} \mid \hat{1}_{n}^{\prime}\right\rangle\left(\varphi_{n}^{a}\right) \sigma_{\mu_{n}^{\prime} \theta_{n}^{\prime}}\left(d \varphi_{n}^{a}\right)\right\}<\infty,
$$

where $d$ is the Hellinger distance.

Proof. The necessity is obvious. For the sufficiency, we first notice that the incomplete direct products with different reference vectors are isomorphic to each other. Thus $\sigma_{\mu, \theta} \cong \sigma_{\mu^{\prime}, \theta^{\prime}}$ implies that $\operatorname{dim} H_{\mu, \theta}\left(\varphi^{a}\right)=\operatorname{dim} H_{\mu^{\prime}, \theta^{\prime}}\left(\varphi^{a}\right)$ for $\sigma_{\mu, \theta}$-a.e. $\varphi^{a}$ and the conclusion follows from Theorem 2.5.

As for the second half, " $\sigma_{\mu, \theta} \cong \sigma_{\mu^{r}, \theta^{\prime}}$ " is equivalent to

$$
\sum_{n=1}^{\infty} d^{2}\left\{\rho_{n}\left(\varphi_{n}^{a}\right) \sigma_{\mu_{n}, \theta_{n}}\left(d \varphi_{n}^{a}\right), \rho_{n}^{\prime}\left(\varphi_{n}^{a}\right) \sigma_{\mu_{n}^{\prime}, \theta_{n}}\left(d \varphi_{n}^{a}\right)\right\}<\infty
$$

by virtue of Kakutani's theorem. (See, [5].) On the other hand, by the choice of $\rho_{n}, \rho_{n}^{\prime}$ it holds that

$$
\begin{aligned}
& \sum_{n=1}^{\infty} d^{2}\left\{\left\langle\hat{1}_{n} \mid \hat{1}_{n}\right\rangle\left(\varphi_{n}^{a}\right) \sigma_{\mu_{n}, \theta_{n}}\left(d \varphi_{n}^{a}\right), \rho_{n}\left(\varphi_{n}^{a}\right) \sigma_{\mu_{n}, \theta_{n}}\left(d \varphi_{n}^{a}\right)\right\}<\infty \\
& \sum_{n=1}^{\infty} d^{2}\left\{\left\langle\hat{1}_{n}^{\prime} \mid \hat{1}_{n}^{\prime}\right\rangle\left(\varphi_{n}^{a}\right), \sigma_{\mu_{n}^{\prime} \theta_{n}^{\prime}}\left(d \varphi_{n}^{a}\right), \rho_{n}^{\prime}\left(\varphi_{n}^{a}\right) \sigma_{\mu_{n}^{\prime}, \theta_{n}^{\prime}}\left(d \varphi_{n}^{a}\right)\right\}<\infty .
\end{aligned}
$$

Therefore (5.15) is equivalent to (5.16).

Example 4. Consider $\boldsymbol{R}^{n_{k}}$-quasi-invariant measures $\mu_{k}$ on $\left(\boldsymbol{R}^{n_{k}}, \mathfrak{B}\left(\boldsymbol{R}^{n_{k}}\right)\right)$ and 1-cocycles $\theta_{k}$ such that $\theta_{k}(x, t)=q_{k}(x-t) q_{k}(x)^{-1}$. Then as we have seen in Example 1, $\left(V_{\mu_{k}, \theta_{k}}, \boldsymbol{R}^{n_{k}}\right)$ has multiplicity 1 and $\left\langle\hat{1}_{k} \mid \hat{1}_{k}\right\rangle(y) \sigma_{\mu_{k}, \theta_{k}}(d y)=$ $\left|\mathcal{F}\left(q_{k} \sqrt{\rho_{k}}\right)\right|^{2}(y) d y$, where $\rho_{k}$ is the density of $\mu_{k}$ with respect to the Lebesgue measure. Thus their product representation $V_{\mu, \theta}$ is cyclic, and $\left(V_{\mu, \theta}, \boldsymbol{R}_{0}^{\infty}\right)$ is equivalent to $\left(V_{\mu^{\prime}, \theta^{\prime}}, \boldsymbol{R}_{0}^{\infty}\right)$ if and only if

$$
\sum_{k=1}^{\infty}\left\{1-\int_{\boldsymbol{R}^{n_{k}}}\left|\mathcal{F}\left(q_{k} \sqrt{\rho_{k}}\right)\right|(y)\left|\mathcal{F}\left(q_{k}^{\prime} \sqrt{\rho_{k}^{\prime}}\right)\right|(y) d y\right\}<\infty .
$$

Here we shall make addition to the ergodicity of a product measure, as we have done in 5.1.

Theorem 5.6. Under the notation in this subsection, $\mu$ is $\Phi$-ergodic if and

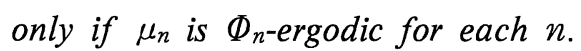


Proof. The necessity is obvious. For the sufficiency, let $A \in \mathfrak{B}$ such that $\mu((A-\varphi) \ominus A)=0$ for all $\varphi \in \Phi$. By the definition of $\mathfrak{B}$, there exist $x_{n, j}^{*} \in X_{n}^{*}(n$ $\left.=1, \cdots, k_{j}, j=1, \cdots\right)$ such that $A$ belongs to the minimal $\sigma$-algebra $\mathbb{E}$ with which all the functions $x \in X \longmapsto \sum_{n=1}^{k_{j}}\left\langle x_{n}, x_{n, j}^{*}\right\rangle(j=1, \cdots)$ are measurable. Let us denote the minimal $\sigma$-algebra on $\prod_{n=1}^{N} X_{n}$ with which all the functions $\left(x_{1}, \cdots\right.$, $\left.x_{N}\right) \longmapsto \sum_{n=1}^{\min \left(N, k_{j}\right)}\left\langle x_{n}, x_{n, j}^{*}\right\rangle$ are measurable by $\mathfrak{\complement}_{N}$. Then we have $A^{y} \in \mathfrak{c}_{N}$ for

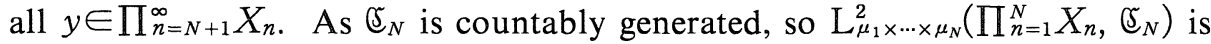
separable. Hence $\left(\Phi_{1} \times \cdots \times \Phi_{N}, d_{N}\right)$ is separable for the total variation metric $d_{N}$ derived from $\mu_{1} \times \cdots \times \mu_{N} \mid \mathbb{E}_{N}$. Choose a countable dense set $\left\{\varphi_{N, k}\right\}_{k}$ from $\left(\Phi_{1}\right.$ $\left.\times \cdots \times \Phi_{N}, d_{N}\right)$, and put $\mu^{1}:=\mu_{1} \times \cdots \times \mu_{N}$ and $\mu^{2}:=\mu_{N+1} \times \cdots \times \mu_{M} \times \cdots$. Then it follows from Fubini's theorem that there exists some $\mu^{2}$-negligible set $\Omega^{c}$ such that for all $y \in \Omega$

$$
\mu^{1}\left(\left(A^{y}-\varphi_{N, k}\right) \ominus A^{y}\right)=0
$$

for all $k$. Hence proceeding in the same way as before, we have for all $y \in \Omega$

$$
\mu^{1}\left(\left(A^{y}-\varphi\right) \ominus A^{y}\right)=0
$$

for all $\varphi \in \Phi_{1} \times \cdots \times \Phi_{N}$. Since $\mu^{1}$ is ergodic due to Theorem 5.2, so $\mu^{1}\left(A^{y}\right)=1$ or 0 for $\mu^{2}$-a.e. $y$. Now for any $\epsilon>0$ there exists a tame set $A_{\epsilon}=\left\{x \in X \mid\left(x_{1}, \cdots\right.\right.$, $\left.\left.x_{N}\right) \in B_{\epsilon}\right\}$ such that $\mu\left(A \ominus A_{\epsilon}\right)<\epsilon$. So

$$
\begin{aligned}
\mu(A) \mu\left(A_{\epsilon}^{c}\right)+\mu\left(A_{\epsilon}\right) \mu\left(A^{c}\right) & =\int_{\prod_{N+1}^{\infty} X_{n}}\left\{\mu^{1}\left(B_{\epsilon}^{c}\right) \mu^{1}\left(A^{y}\right)+\mu^{1}\left(\left(A^{y}\right)^{c}\right) \mu^{1}\left(B_{\epsilon}\right)\right\} \mu_{2}(d y) \\
& =\mu\left(A \ominus A_{\epsilon}\right)<\epsilon .
\end{aligned}
$$

As $\epsilon$ is arbitrary, $\mu(A) \mu\left(A^{c}\right)=0$.

\section{§6. Gaussian Measure}

6.1 Gaussian measure. Let $\gamma$ be a Gaussian measure on $(X, \mathfrak{B})$. That is, its characteristic function $\bar{\gamma}\left(x^{*}\right), x^{*} \in X^{*}$ has the following form.

$$
\hat{\gamma}\left(x^{*}\right)=\exp \left(i m\left(x^{*}\right)-2^{-1} v^{2}\left(x^{*}\right)\right),
$$

where

$$
m\left(x^{*}\right)=\int_{X}\left\langle x, x^{*}\right\rangle \gamma(d x) \text { and } v^{2}\left(x^{*}\right)=\int_{X}\left\{\left\langle x, x^{*}\right\rangle-m\left(x^{*}\right)\right\}^{2} \gamma(d x) .
$$

Put $\bar{\gamma}(E):=\gamma(-E)$ for all $E \in \mathfrak{B}$ and let $g$ be a image measure of the convolution of $\gamma * \bar{\gamma}$ by the homothety, $x \longmapsto 2^{-1 / 2} x$. Then

$$
\widehat{g}\left(x^{*}\right)=\hat{\gamma}\left(2^{-1 / 2} x^{*}\right) \hat{\bar{\gamma}}\left(2^{-1 / 2} x^{*}\right)=\exp \left(-2^{-1} v^{2}\left(x^{*}\right)\right)
$$

and 


$$
\widehat{\gamma}\left(x^{*}\right)=\exp \left(i m\left(x^{*}\right)\right) \hat{g}\left(x^{*}\right) .
$$

In this section we shall consider representations such type as

$$
V_{\gamma, s}(\varphi): h(x) \in \mathrm{L}_{\gamma}^{2} \longmapsto\left(\frac{d \gamma_{\varphi}}{d \gamma}\right)^{1 / 2+i s} h(x-\varphi) \in \mathrm{L}_{\gamma}^{2}
$$

where $\varphi \in A_{\gamma}^{\circ}$ and $s$ is a real parameter.

Now consider a map $M$ defined for the tame bounded functions such that

$M: f\left(\left\langle x, x_{1}^{*}\right\rangle, \cdots,\left\langle x, x_{n}^{*}\right\rangle\right) \in \mathrm{L}_{g}^{2} \longmapsto f\left(\left\langle x, x_{1}^{*}\right\rangle-m\left(x_{1}^{*}\right), \cdots,\left\langle x, x_{n}^{*}\right\rangle-m\left(x_{n}^{*}\right)\right) \in$ $\mathrm{L}_{\gamma}^{2}$.

It is easy to see that $\mathcal{M}$ is well defined and has a unitary extension which will be denoted by the same letter. Put

$$
S_{\varphi}:=M\left(\sqrt{\frac{d g_{\varphi}}{d g}}\right)
$$

for $\varphi \in A_{g}^{\circ}$. Then $s_{\varphi}(x) \geq 0$ for $\gamma$-a.e. $x$ and

$$
\begin{aligned}
& \int_{X} S_{\varphi}^{2}(x) \chi_{E}\left(\left\langle x, x_{1}^{*}\right\rangle, \cdots,\left\langle x, x_{n}^{*}\right\rangle\right) \gamma(d x) \\
& =\int_{X} \chi_{E}\left(\left\langle x, x_{1}^{*}\right\rangle+m_{1}\left(x^{*}\right), \cdots,\left\langle x, x_{n}^{*}\right\rangle+m_{n}\left(x^{*}\right)\right) g_{\varphi}(d x) \\
& =\int_{X} \chi_{E}\left(\left\langle x, x_{1}^{*}\right\rangle, \cdots,\left\langle x, x_{n}^{*}\right\rangle\right) \gamma_{\varphi}(d x) .
\end{aligned}
$$

It derives that

$$
\gamma_{\varphi} \cong \gamma \text { and } \sqrt{\frac{d \gamma_{\varphi}}{d \gamma}}=M\left(\sqrt{\frac{d g_{\varphi}}{d g}}\right)
$$

for $\varphi \in A_{g}^{\circ}$. In a similar way, we have

$$
A_{\gamma}^{\circ}=A_{g}^{\circ}
$$

Note that $\mathcal{M}(h \cdot f)=\mathcal{M}(h) \cdot \mathcal{M}(f)$ for all $h \in \mathrm{L}_{g}^{2}$ and for all $f \in \mathrm{L}_{g}^{\infty}$. Thus we have

$$
M\left(\left(\frac{d \gamma_{\varphi}}{d \gamma}\right)^{1 / 2+s i}\right)=\left(\frac{d g_{\varphi}}{d g}\right)^{1 / 2+s i},
$$

and it follows that

$$
\begin{aligned}
& M \circ V_{g, s}(\varphi)(f)(x)=\mathcal{M}\left(\left(\frac{d g_{\varphi}}{d g}(\cdot)\right)^{1 / 2+s i} f(\cdot-\varphi)\right)(x) \\
& =\left(\frac{d \gamma_{s}}{d \gamma}\right)^{1 / 2+s i}(x)(M f)(x-\varphi)=V_{\gamma, s}(\varphi) \circ \mathcal{M}(f)(x) .
\end{aligned}
$$

Theorem 6.1. $\left(V_{\gamma, s}, A_{\gamma}^{\circ}\right)$ is equivalent to $\left(V_{g, s}, A_{g}^{\circ}\right)$ by the intertwining 
operator M. Moreover $\gamma$ is $\Phi$-ergodic if and only if so is $g$.

Proof. We only have to show the second assertion. Suppose that $\gamma$ is $\Phi$-ergodic and $g((B-\varphi) \ominus B)=0$ for all $\varphi \in \Phi$. Then as $M\left(\chi_{E}(\cdot-\varphi)\right)(x)=$ $\left(M \chi_{E}\right)(x-\varphi)$, which is first valid for the tame set $E$ and generally holds by limiting procedure, so $M \chi_{B}=$ const $\bmod \gamma$ and we have $\chi_{B}=1$ or $0 \bmod g$. The converse will be proved similarly.

By the above theorem it is sufficient to consider only centered Gaussian measures $g$ for our subject. Now set

$$
\left\|x^{*}\right\|_{g}:=\left\{\int_{X}\left\langle x, x^{*}\right\rangle^{2} g(d x)\right\}^{1 / 2}
$$

for $x^{*} \in X^{*}$, and put

$$
H_{g}:=\left\{\varphi \in X\left\|\left\langle\varphi, x^{*}\right\rangle \mid \leq{ }^{\exists} C_{\varphi}\right\| x^{*} \|_{g}\right\},
$$

which is called the reproducing kernel Hilbert space. Then for any $\varphi \in H_{g}$ there exists a unique $W_{\varphi}(x)$ belonging to the $L^{2}$-closure $W$ of $\left\{\left\langle x, x^{*}\right\rangle \mid x^{*} \in X^{*}\right\}$ such that

$$
\left\langle\varphi, x^{*}\right\rangle=\int_{X}\left\langle x, x^{*}\right\rangle W_{\varphi}(x) g(d x)
$$

Since a map $\varphi \longrightarrow W_{\varphi}$ is one to one, so an inner product structure is naturally induced from $\mathrm{L}_{g}^{2}$. Moreover it is well known that

$$
\begin{gathered}
A_{g}^{\circ}=H_{g} \\
\frac{d g_{\varphi}}{d g}(x)=\exp \left(W_{\varphi}(x)-2^{-1}\|\varphi\|_{g}^{2}\right) \text { and } \\
W_{\varphi}(x+h)=W_{\varphi}(x)+\langle\varphi, h\rangle_{H g}
\end{gathered}
$$

for all $\varphi, h \in H_{g}$. As for the Kakutani's metric $d_{g}$ on $A_{\mu}^{\circ}$, we have

$$
d_{g}(\varphi, 0)^{2}=2\left\{1-\exp \left(-8^{-1}\|\varphi\|_{H_{g}}^{2}\right)\right\}
$$

Thus the topologies on $H_{g}$ derived from $d_{g}$ and the norm $\|\cdot\|_{H_{g}}$ coincide and it is stronger than $\sigma\left(X, X^{*}\right)$. As we assume that $\mathrm{L}_{g}^{2}$ is separable, so is $H_{g}$.

Proposition 6.1. The followings are all equivalent.

(1) $g$ is $H_{g}$-ergodic.

(2) The $\mathrm{L}^{2}$-closure $W^{\prime}$ of $\left\{W_{\varphi}\right\}_{\varphi \in H_{g}}$ coincides with $W$.

(3) There exists an orthonormal set $\left\{h_{n}\right\}_{n} \subset H_{g}$ such that 


$$
\left\langle x, x^{*}\right\rangle=\sum_{n=1}^{\infty}\left\langle h_{n}, x^{*}\right\rangle W_{h n}(x)
$$

for all $x^{*} \in X^{*}$, where the equality holds in $\mathrm{L}_{g}^{2}$-sense.

Proof. $\quad(1) \Rightarrow(2) . \quad$ Take any $S(x) \in W \cap\left(W^{\prime}\right)^{\perp}$. Then there exists a sequence $\left\{x_{n}^{*}\right\}_{n} \subset X^{*}$ such that $\left\langle x, x_{n}^{*}\right\rangle \longrightarrow S(x)(n \longrightarrow \infty)$ in $\mathrm{L}_{g}^{2}$. Since

$$
\lim _{n \rightarrow \infty}\left\langle\varphi, x_{n}^{*}\right\rangle=\lim _{n \rightarrow \infty} \int_{X}\left\langle x, x_{n}^{*}\right\rangle W_{\varphi}(x) g(d x)=\int_{X} S(x) W_{\varphi}(x) g(d x)=0,
$$

so, if necessary, taking a subsequence $\left\{x_{n}^{*}\right\}_{n}$ we have

$$
S(x+\varphi)=\lim _{n \rightarrow \infty}\left\langle x+\varphi, x_{n}^{*}\right\rangle=S(x)
$$

for $g$-a.e.x. Thus $S(x)=$ const $\bmod g$ and the constant is equal to 0 because $\int_{X} S(x) g(d x)=0$.

(2) $\Rightarrow(3)$. Let $\left\{h_{n}\right\}_{n} \subset H_{g}$ be a complete orthonormal set in the completion on $H_{g}$. By the assumption $\left\langle x, x^{*}\right\rangle$ is adherent to the linear span of $W_{h_{n}}^{\prime}$ s. Therefore (6.17) exactly holds by virtue of (6.12).

$(3) \Rightarrow(1)$. Suppose that $g((B-\varphi) \ominus B)=0$ for all $\varphi \in H_{g}$. By the assumption there exists a measurable set $\widehat{B} \subseteq \boldsymbol{R}^{\infty}$ such that $\chi_{B}(x)=\chi_{\bar{B}}\left(\left(W_{h_{1}}(x), \cdots, W_{h_{n}}(x)\right.\right.$, $\cdots))$. Now take any $\left(\alpha_{1}, \cdots, \alpha_{n}\right) \in \boldsymbol{R}^{n}$ and set $\varphi:=\alpha_{1} h_{1}+\cdots+\alpha_{n} h_{n}$. As the image of $g$ by a map $x \in X \longrightarrow\left(W_{h_{1}}(x), \cdots, W_{h_{n}}(x), \cdots\right) \in \boldsymbol{R}^{\infty}$ is the standard Gaussian measure $G$ on $\boldsymbol{R}^{\infty}$, so

$$
\begin{aligned}
& \int_{R^{\infty}}\left|\chi_{\bar{B}}\left(y+\left(\alpha_{1}, \cdots, \alpha_{n}, 0,0, \cdots\right)\right)-\chi_{\hat{B}}(y)\right| G(d y) \\
& =\int_{X} \mid \chi_{\hat{B}}\left(\left(W_{h_{n}}(x+\varphi)\right)-\chi_{\bar{B}}\left(\left(W_{h_{n}}(x)\right) \mid g(d x)\right.\right. \\
& =\int_{X}\left|\chi_{B}(x+\varphi)-\chi_{B}(x)\right| g(d x)=0 .
\end{aligned}
$$

It follows that $G(\widehat{B})=1$ or 0 , because $G$ is $\boldsymbol{R}_{0}^{\infty}$-ergodic. So, $g(B)=1$ or 0 .

Remark. We don't yet know whether the conditions of Proposition 6.1 always hold or not, and further $H_{g}$ is always complete or not. However if $\gamma$ is extended to a weak Radon measure on $X$, (the extension is unique,) then the above questions are all solved affirmatively. Besides, it holds certainly that $m$ $\in\left(X^{*}\right)^{a}$ in (6.2) belongs to $X$. Moreover provided that $X$ is $\sigma\left(X, X^{*}\right)$ complete, then $H_{g}$ is complete with or without Radon extensibility, which is easily seen by virtue of (P.4).

Proposition 6.2. Let $\widetilde{X}$ be the completion of $\left(X, \sigma\left(X, X^{*}\right)\right)$ and put $\widetilde{g}$ be the image measure of $g$ by the imbedding map $\iota: X \longmapsto \widetilde{X}$. Then if $g$ 
is $H_{g}$-ergodic, then so is $\widetilde{g}$, and $H_{g}$ is dense in $H_{\tilde{g}}$.

Proof. It is easy to see that $H_{g} \subseteq H_{\widetilde{g}}$ and $\widetilde{g}$ is $H_{g}$-ergodic, and that the norm on $H_{\widetilde{g}}$ is an extension of the norm on $H_{g}$. We shall prove that $H_{g}$ is dense in $H_{\tilde{g}}$. Suppose that it would be false. Then there exists some $\widetilde{S}(\widetilde{x})(\neq 0)$ belonging to the $\mathrm{L}^{2}$-closure of $\left\{\left\langle\tilde{x}, x^{*}\right\rangle\right\}_{x^{*} \in X^{*}}$ such that $\int_{\tilde{X}} \widetilde{S}(\tilde{x}) \widetilde{W}_{h}(\tilde{x}) \widetilde{g}(d \tilde{x})$ $=0$ for all $h \in H_{g}$. Thus proceeding in the same way as before, we have $\widetilde{S}(\tilde{x}$ $+h)=\widetilde{S}(\widetilde{x})$ for $\widetilde{g}$-a.e. $\widetilde{x}$. It follows that $\widetilde{S}(\tilde{x})=0$ for $\widetilde{g}$-a.e. $\widetilde{x}$, which contradicts to the assumption.

In a little while, we shall take and fix a complete orthonormal set $\left\{h_{n}\right\}_{n} \subset H_{g}$ in the completion of $H_{g}$ and $\left\{\omega_{q}\right\}_{q \in Q}$ which is a c.o.n.s. in $W \cap\left(W^{\prime}\right)^{\perp}$. As we have seen,

$$
\left\langle x, x^{*}\right\rangle=\sum_{n=1}^{\infty}\left\langle h_{n}, x^{*}\right\rangle W_{h_{n}}(x)+\sum_{q \in Q} \alpha_{q} \omega_{q}(x),
$$

where $\alpha_{q}=\int_{X}\left\langle x, x^{*}\right\rangle \omega_{q}(x) g(d x)$, and it holds that for all $\varphi \in H_{g}$

$$
\omega_{q}(x+\varphi)=\omega_{q}(x)
$$

for $g$-a.e.x. Now we shall find a direct integral for the representation $\left(V_{g, s}, H_{g}\right)$. So let us take tame functions $h$ and $g$ such that $h(x)=H\left(W_{h_{1}}(x), \cdots, W_{h_{N}}(x)\right.$, $\left.\omega_{1}(x), \cdots, \omega_{M}(x)\right), g(x)=G\left(W_{h_{1}}(x), \cdots, W_{h_{N}}(x), \omega_{1}(x), \cdots, \omega_{M}(x)\right)$.

We calculate $\left\langle V_{g, s}(\varphi) h, g\right\rangle_{2}$ for $\varphi=\sum_{n=1}^{\infty} \varphi_{n} h_{n} \in H_{g}$.

$$
\begin{aligned}
\langle & \left.V_{g, s}(\varphi) h, g\right\rangle_{2} \\
= & \int_{X}\left(\frac{d g_{\varphi}}{d g}\right)^{1 / 2+s i} h(x-\varphi) \bar{g}(x) g(d x) \\
= & \exp \left(-2^{-1}\left(2^{-1}+s i\right)\|\varphi\|_{H g}^{2}\right) \int_{X} \exp \left(\left(2^{-1}+s i\right) W_{\varphi}(x)\right) H\left(W_{h_{1}}(x)-\varphi_{1}, \cdots, W_{h_{N}}(x)\right. \\
& \left.-\varphi_{N}, \omega_{1}(x), \cdots, \omega_{M}(x)\right) \bar{G}\left(W_{h_{1}}(x), \cdots, W_{h_{N}}(x), \omega_{1}(x), \cdots, \omega_{M}(x)\right) g(d x) \\
= & \exp \left(-2^{-1}\left(2^{-1}+s i\right)\|\varphi\|_{H g}^{2}\right) \exp \left(2^{-1}\left(2^{-1}+s i\right)^{2} \sum_{n=N+1}^{\infty} \varphi_{n}^{2}\right) \cdot \\
& \int_{R^{N}} \int_{R^{M}} \exp \left(\left(2^{-1}+s i\right) \sum_{n=1}^{N} \varphi_{n} x_{n}\right) \cdot \\
& H\left(x_{1}-\varphi_{1}, \cdots, x_{N}-\varphi_{N}, y_{1}, \cdots, y_{M}\right) \bar{G}\left(x_{1}, \cdots, x_{N}, y_{1}, \cdots, y_{M}\right) g_{N}(d x) g_{M}(d y),
\end{aligned}
$$

where $g_{N}$ is the standard Gaussian measure on $\boldsymbol{R}^{N}$. And

$$
\begin{aligned}
\langle & \left.V_{g, s}(\varphi) h, g\right\rangle_{2} \\
= & \exp \left(-8^{-1}\left(1+4 s^{2}\right) \sum_{n=N+1}^{\infty} \varphi_{n}^{2}\right) \int_{R^{N}} \int_{R^{M}}(2 \pi)^{-N / 2} \exp \left(-2^{-1}\left(2^{-1}+s i\right)\right. \\
& \left.\sum_{n=1}^{N}\left(x_{n}-\varphi_{n}\right)^{2}\right) H\left(x_{1}-\varphi_{1}, \cdots, x_{N}-\varphi_{N}, y_{1}, \cdots, y_{M}\right) \exp \left(-2^{-1}\left(2^{-1}-s i\right) \sum_{n=1}^{N} x_{n}^{2}\right) 。
\end{aligned}
$$




$$
\bar{G}\left(x_{1}, \cdots, x_{N}, y_{1}, \cdots, y_{M}\right) g_{M}(d y) d x .
$$

Consequently,

$$
\begin{aligned}
& \left\langle V_{g, s}(\varphi) h, g\right\rangle_{2} \\
& =\exp \left(-8^{-1}\left(1+4 s^{2}\right) \sum_{n=N+1}^{\infty} \varphi_{n}^{2}\right) \int_{R^{N}} \int_{R^{\mu}} \exp \left(i \sum_{n=1}^{N} \varphi_{n} x_{n}\right) \\
& \mathcal{F}\left\{(2 \pi)^{-N / 4} \exp \left(-2^{-1}\left(2^{-1}+s i\right) \sum_{n=1}^{N} \xi_{n}^{2}\right) \cdot\right. \\
& H(\xi, y)\}(x) \bar{F}\left\{(2 \pi)^{-N / 4} \exp \left(-2^{-1}\left(2^{-1}+s i\right) \sum_{n=1}^{N} \xi_{n}^{2}\right) K(\xi, y)\right\}(x) d x g_{M}(d y) .
\end{aligned}
$$

Now consider a measure $m_{H}$ whose density is given by

(6.21) $\frac{m_{H}(d x)}{d x}=\int_{R^{M}}\left|\mathcal{F}\left\{(2 \pi)^{-N / 4} \exp \left(-2^{-1}\left(2^{-1}+s i\right) \sum_{n=1}^{N} \xi_{n}^{2}\right) H(\xi, y)\right\}\right|^{2}(x) \gamma_{M}(d y)$.

Then (6.20) is rewritten as

$$
\left\langle V_{g, s}(\varphi) h, h\right\rangle_{2}=\exp \left(-8^{-1}\left(1+4 s^{2}\right) \sum_{n=N+1}^{\infty} \varphi_{n}^{2}\right) \widehat{m}_{H}\left(\left(\varphi_{1}, \cdots, \varphi_{N}\right)\right) .
$$

Here let us take a Gaussian measure $G_{s}$ on $\left(H^{a}, \widetilde{C}_{H}\right)$ such that $\widehat{G}_{s}(\varphi)=$ $\exp \left(-8^{-1}\left(1+4 s^{2}\right)\|\varphi\|_{H_{g}}^{2}\right)$ and put $P_{N}(\varphi):=\sum_{n=1}^{N} \varphi_{n} h_{n}, Q_{N}:=\mathrm{I}-P_{N}$ and $h_{n}^{*}(\varphi)$ $:=\left\langle\varphi, h_{n}\right\rangle_{H_{g}}$. Using an isomorphism $\eta$ from $H^{a}$ to $\boldsymbol{R}^{N} \times\left\{h_{1}, \cdots, h_{N}\right\}^{\perp}$ such that

$$
\eta: \varphi^{a} \longmapsto\left(\left(\left\langle h_{1}, \varphi^{a}\right\rangle, \cdots,\left\langle h_{N}, \varphi^{a}\right\rangle\right), \varphi^{a}-\sum_{n=1}^{N}\left\langle h_{n}, \varphi^{a}\right\rangle h_{n}^{*}\right),
$$

we obtain an image measure $\eta^{-1}\left(m_{H} \times{ }^{t} Q_{N} G_{s}\right)$ on $H^{a}$. It is quite easy to see that its characteristic function coincides with $\left\langle V_{g, s}(\varphi) h, h\right\rangle_{2}$. Further $m_{1}$ (corresponding to $H=1$ ) is equivalent to the Lebesgue measure $\lambda_{N}$ on $\boldsymbol{R}^{N}$, so $m_{H} \times$ ${ }^{t} Q_{N} G_{s}$ is absolutely continuous with $m_{1} \times{ }^{t} Q_{N} G_{s}$ and $m_{1} \times{ }^{t} Q_{N} G_{s}$ correspondes to $G_{s}$ by the above map $\eta$. It gives that $G_{s}$ is a spectral measure for $\left(V_{g, s}, H_{g}\right)$. (It is realized as $H=1$.)

Now let us consider the multiplicity. We shall rewrite (6.20) as the following form using the spectral measure $G_{S}$ and the standard Gaussian measure $g_{Q}$ on $\boldsymbol{R}^{Q}$.

$$
\begin{aligned}
&\left\langle V_{g, s}(\varphi) h, g\right\rangle_{2} \\
&=\int_{H^{a}} \int_{R^{Q}} \exp \left(i\left\langle\varphi, \varphi^{a}\right\rangle\right)\left\{(2 \pi)^{-1 / 4} \sqrt{\frac{2}{1+2 s i}}\right\}^{-N} \exp \left(\frac{1}{1+2 s i} \sum_{n=1}^{N}\left\langle h_{n}, \varphi^{a}\right\rangle^{2}\right) . \\
& \mathcal{F}\left\{(2 \pi)^{-N / 4} \exp \left(-2^{-1}\left(2^{-1}+s i\right) \sum_{n=1}^{N} \xi_{n}^{2}\right) H(\xi, y)\right\}\left(\left\langle h_{1}, \varphi^{a}\right\rangle, \cdots,\left\langle h_{N}, \varphi^{a}\right\rangle\right) . \\
&\left\{(2 \pi)^{-1 / 4} \sqrt{\frac{2}{1-2 s i}}\right\}^{-N} \exp \left(\frac{1}{1-2 s i} \sum_{n=1}^{N}\left\langle h_{n}, \varphi^{a}\right\rangle^{2}\right) .
\end{aligned}
$$




$$
\begin{aligned}
& \bar{F}\left\{(2 \pi)^{-N / 4} \exp \left(-2^{-1}\left(2^{-1}+s i\right) \sum_{n=1}^{N} \xi_{n}^{2}\right) \cdot\right. \\
& G(\xi, y)\}\left(\left\langle h_{1}, \varphi^{a}\right\rangle, \cdots,\left\langle h_{N}, \varphi^{a}\right\rangle\right) G_{s}\left(d \varphi^{a}\right) g_{Q}(d y) .
\end{aligned}
$$

Hence a map

$$
\begin{aligned}
& S_{s}: H\left(W_{h_{1}}(x), \cdots, W_{h_{N}}(x), \omega_{1}(x), \cdots, \omega_{M}(x)\right) \\
& \longmapsto\left\{(2 \pi)^{-1 / 4} \sqrt{\frac{2}{1+2 s i}}\right\}^{-N} \exp \left(\frac{1}{1+2 s i} \sum_{n=1}^{N}\left\langle h_{n}, \varphi^{a}\right\rangle^{2}\right) \mathscr{F}\left\{(2 \pi)^{-N / 4}\right. \\
&\left.\exp \left(-2^{-1}\left(2^{-1}+s i\right) \sum_{n=1}^{N} \xi_{n}^{2}\right) H\left(\xi_{1}, \cdots, \xi_{N}, y_{1}, \cdots, y_{M}\right)\right\}
\end{aligned}
$$

is well defined for the tame functions $h$ and it has a unitary extension from $\mathrm{L}_{g}^{2}$ to $\mathrm{L}_{G s}^{2}\left(H^{a}, \mathrm{~L}_{g_{Q}}^{2}\left(\boldsymbol{R}^{Q}\right)\right)$ of all $\mathrm{L}_{g_{Q}}^{2}\left(\boldsymbol{R}^{Q}\right)$-valued square summable functions.

(The onto property follows from

$$
\left.S_{s}\left(\left(\frac{d g_{\varphi}}{d g}\right)^{1 / 2+s i} h(x-\varphi)\right)\left(\varphi^{a}\right)=\exp \left(i\left\langle\varphi, \varphi^{a}\right\rangle\right)\left(S_{s} h\right)\left(\varphi^{a}\right) .\right)
$$

And we have

$$
\int_{X}\left(\frac{d g_{\varphi}}{d g}\right)^{1 / 2+s i} h(x-\varphi) g(x) g(d x)=\int_{H^{a}} \exp \left(i\left\langle\varphi, \varphi^{a}\right\rangle\right)\left\langle S_{s} h \mid S_{s} g\right\rangle_{\mathrm{L}_{g_{Q}}^{2}}\left(\varphi^{a}\right) G_{s}\left(d \varphi^{a}\right)
$$

We settle these arguments as the following theorem.

Theorem 6.2. Let $g$ be a centered Gaussian measure on $(X, \mathfrak{B})$ and $H_{g}$ be the reproducing kernel space. Then the spectral measure $G_{s}$ for $\left(V_{g, s}, H_{g}\right)$ is given by

$$
\widehat{G}_{s}(\varphi)=\exp \left(-8^{-1}\left(1+4 c^{2}\right)\|\varphi\|_{H g}^{2}\right)=\int_{X}\left(\frac{d g_{\varphi}}{d g}\right)^{1 / 2+s i}(x) g(d x) .
$$

Further the direct integral is realized as $L_{G_{s}}^{2} \otimes L_{R^{\circ}}^{2}$ and the corresponding map from $L_{g}^{2}$ to $L_{G_{s}}^{2} \otimes L_{R^{\circ}}^{2}$ is given by (6.23). Therefore it has uniform mutiplicity 1 or $\infty$ according to whether $g$ is H-ergodic or not.

Corollary 6.3. Let $g_{s}$ be the image measure of $g$ by the homothety, $x$ $\longmapsto\left(1+4 s^{2}\right)^{-1 / 2} x$. Then the representation $\left(V_{g, s}, H_{g}\right)$ is equivalent to $\left(V_{g_{s}, 0}\right.$, $\left.H_{g_{s}}\right)$.

Proof. It is obvious that $A_{g}^{\circ}=A_{g_{s}}^{\circ}$ and the ergodic notion is invariant for these measures. Thus two representations have the same multiplicity. Let $\lambda_{g_{s}, 0}$ be the spectral measure of $\left(V_{g_{s}, 0}, H_{g_{s}}\right)$. Then $\hat{\lambda}_{g_{s}, 0}(\varphi)=\exp \left(-8^{-1}\|\varphi\|_{H_{g_{s}}}^{2}\right)$ and 


$$
\|\varphi\|_{H_{g_{s}}}=\sup _{\left\|x^{*}\right\|_{g_{s}}=1}\left\langle\varphi, x^{*}\right\rangle=\sup _{\left\|x^{*}\right\|_{g}=\sqrt{1+4 s^{2}}}\left\langle\varphi, x^{*}\right\rangle=\sqrt{1+4 s^{2}}\|\varphi\|_{H g}
$$

Thus they have the same spectral measure.

Remark. For the case $g$ is $H_{g}$-ergodic, $S_{s}$ is regarded as a kind of Fourie transform on infinite dimensional spaces. Without proofs we shall give an interesting formula such that

$$
\left\{\begin{array}{c}
S_{s} \circ V_{g, s}(\varphi) \circ S_{s}^{-1} \widetilde{J}\left(\varphi^{a}\right)=\exp \left(i\left\langle\varphi, \varphi^{a}\right\rangle\right) \widetilde{J}\left(\varphi^{a}\right) \\
S_{s} \circ U_{g}\left(x^{*}\right) \circ S_{s}^{-1} \widetilde{J}\left(\varphi^{a}\right)\left(\frac{d\left(G_{s}\right)_{-x^{*}}}{d G_{s}}\right)^{1 / 2-s i} \widetilde{J}\left(\varphi^{a}+x^{*}\right)
\end{array}\right.
$$

for all $\widetilde{J} \in L_{\lambda g, s}^{2}$. That is $s$ is changed to $-s$ under the dual operation. Besides, $g$ and $G_{s}$ correspondes to the norms of the same type, if $s=\sqrt{3} / 2$.

Theorem 6.4. Let $s^{\prime}$ be a real number and $g^{\prime}$ be another centered Gaussian measure on $(X, \mathfrak{B})$ such that $A_{g}^{\circ}=A_{g^{\prime}}^{\circ}=: E$.

(1) If the representations $\left(V_{g, s}, E\right)$ and $\left(V_{g^{\prime}, s^{\prime}}, E\right)$ are equivalent, then both $g$ and $g^{\prime}$ are E-ergodic or so is neither $g$ nor $g^{\prime}$.

(2) If both $g$ and $g^{\prime}$ are E-ergodic or so is neither $g$ nor $g^{\prime}$, then $\left(V_{g, s}, E\right)$ and $\left(V_{g^{\prime}, s^{\prime}}, E\right)$ are equivalent, if and only if the spectral measure $\lambda_{g, s}$ and $\lambda_{g^{\prime}, s^{\prime}}$ are equivalent.

(3) Under an assumption that both $g$ and $g^{\prime}$ are E-ergodic, the representations $\left(V_{g, s}, E\right)$ and $\left(V_{g^{\prime}, s^{\prime}}, E\right)$ are equivalent, if and only if $g_{s}$ is equivalent to $g_{s^{\prime}}$, where $g_{s}$ is the image measure of $g$ by the homothety, $x \longmapsto\left(1+4 s^{2}\right)^{-1 / 2} x$.

Proof. There is nothing to prove (1) and (2) and the sufficiency of (3). In order to prove the necessity of (3), it is sufficient to consider the case $s=s^{\prime}=0$ due to Corollary 6.3. Let $\widetilde{X}$ be the completion of $\left(X, \sigma\left(X, X^{*}\right)\right)$, and $\widetilde{g}, \widetilde{g}^{\prime}$ be the image measures of $g$ and $g^{\prime}$ by the imbedding map $\iota: X \longmapsto \widetilde{X}$, respectively. As it holds that $\mathfrak{B}=\left({ }^{t} \iota\right)^{-1}(\widetilde{\mathfrak{B}})$, where $\widetilde{\mathfrak{B}}$ is the cylindrical $\sigma$-algebra on $\widetilde{X}$, so we only have to check that $\widetilde{g} \cong \widetilde{g}^{\prime}$ for the proof. By the assumption spectral measures are equivalent. Thus the norm $\|\cdot\|_{H g}$ on $E$ is equivalent to $\|\cdot\|_{H^{\prime}}$ and we have

$$
A_{\widetilde{g}}^{\circ}=A_{\widetilde{g}}^{\circ}=: \widetilde{E}
$$

which follows from Proposition 6.2. Now let $G$ and $G^{\prime}$ be the spectral measure on $E^{a}$ for the original representations such that $\widehat{G}(\varphi)=\exp \left(-8^{-1}\|\varphi\|_{H g}^{2}\right)$ and $\widehat{G}^{\prime}(\varphi)=\exp \left(-8^{-1}\|\varphi\|_{H^{\prime}}^{2}\right)$, respectively. Moreover let $\widetilde{G}$ and $\widetilde{G}^{\prime}$ be probability measures on $\widetilde{E}^{a}$ such that $\widehat{\widetilde{G}}(\varphi)=\exp \left(-8^{-1}\|\varphi\|_{H_{\tilde{g}}}^{2}\right)$ and $\widehat{\widetilde{G}}^{\prime}(\varphi)=\exp$ $\left(-8^{-1}\|\varphi\|_{H_{\tilde{g}^{*}}}^{2}\right)$, respectively. Then we have ${ }^{t} \eta \widetilde{G}=G$ and ${ }^{t} \eta \widetilde{G}^{\prime}=G^{\prime}$, where $\eta: E$ 
$\widetilde{E}$ is the imbedding map. Further it is easily checked that $\Im_{\widetilde{E}}=(t \eta)^{-1}\left(\Im_{E}\right)$ $\bmod \widetilde{G}+\widetilde{G}^{\prime}$. Thus $\widetilde{G} \cong \widetilde{G}^{\prime}$ follows from our assumption $G \cong G^{\prime}$. Here we shall use the following well known fact. (For example, see [2] or [11].)

Theorem 6.5. In order that $\widetilde{G} \cong \widetilde{G}^{\prime}$ holds, it is necessary and sufficient that there exists a positive definite isomorphic operator $P$ on $\widetilde{E}$ such that $\mathrm{I}-P$ is a Hilbert-Schmidt type and $\|P \varphi\|_{H_{\tilde{g}}}=\|\varphi\|_{H_{\tilde{g}}}$ for all $\varphi \in \widetilde{E}$.

Let $\tilde{h}_{1}, \cdots, \tilde{h}_{n}, \cdots$, be the complete system of eigen vectors of $P$, and $\left(1+\lambda_{n}\right)$ be the corresponding eigen value. And put

$$
\rho(\tilde{x})=\prod_{n=1}^{\infty}\left(1+\lambda_{n}\right) \exp \left(-2^{-1}\left(2 \lambda_{n}+\lambda_{n}^{2}\right) W_{\widetilde{h}_{n}}^{2}(x)\right) .
$$

The infinite product in (6.27) exactly converges in $\mathrm{L}_{\tilde{g}}^{1}$. We shall calculate the characteristic function of the measure $\rho(\tilde{x}) \widetilde{g}(d \tilde{x})$.

$$
A\left(x^{*}\right):=\int_{\tilde{X}} \exp \left(i\left\langle\tilde{x}, x^{*}\right\rangle\right) \rho(\tilde{x}) \widetilde{g}(d \tilde{x}) .
$$

Since $\widetilde{g}$ is $\widetilde{E}$-ergodic by virtue of Proposition 6.2 , so we have

$$
\left\langle\tilde{x}, x^{*}\right\rangle=\sum_{n=1}^{\infty}\left\langle\tilde{h}_{n}, x^{*}\right\rangle W_{\tilde{h}_{n}}(\tilde{x}) \text { in } \mathrm{L}_{\tilde{g}}^{2} .
$$

Consequently,

$$
\begin{aligned}
A\left(x^{*}\right)=\lim _{N \rightarrow \infty} \int_{\widetilde{x}} \exp \left(i \sum_{n=1}^{N}\left\langle\widetilde{h}_{n}, x^{*}\right\rangle W_{\widetilde{h}_{n}}(\tilde{x})\right) \rho(\tilde{x}) \widetilde{g}(d \tilde{x}) \\
=\lim _{N \rightarrow \infty} \lim _{M \rightarrow \infty} \int_{\widetilde{X}} \exp \left(i \sum_{n=1}^{N}\left\langle\widetilde{h}_{n}, x^{*}\right\rangle W_{\widetilde{h}_{n}}(\tilde{x})\right) \cdot \\
\prod_{n=1}^{M}\left(1+\lambda_{n}\right) \exp \left(2^{-1}\left(2 \lambda_{n}+\lambda_{n}^{2}\right) W_{\widetilde{h}_{n}}^{2}(\tilde{x})\right) \widetilde{g}(d \tilde{x}) \\
=\lim _{N \rightarrow \infty} \lim _{M \rightarrow \infty} \int_{R^{M}} \exp \left(i \sum_{n=1}^{N}\left\langle\tilde{h}_{n}, x^{*}\right\rangle x_{n}\right)(2 \pi)^{-M / 2} \prod_{n=1}^{M}\left(1+\lambda_{n}\right) \exp \left(2^{-1}\left(1+\lambda_{n}\right)^{2} x_{n}^{2}\right) d x \\
=\lim _{N \rightarrow \infty} \exp \left(-2^{-1} \sum_{n=1}^{N}\left(\frac{\left\langle\widetilde{h}_{n}, x^{*}\right\rangle}{1+\lambda_{n}}\right)^{2}\right) .
\end{aligned}
$$

Since $\left\|\left(1+\lambda_{n}\right)^{-1} \widetilde{h}_{n}\right\|_{H_{\widetilde{g}}}=1$ and $\widetilde{g}^{\prime}$ is $\widetilde{E}$-ergodic, so it follows from the corresponding formula to (6.29) to $\widetilde{g}^{\prime}$ that $A\left(x^{*}\right)=\exp \left(-2^{-1}\left\|x^{*}\right\|_{\widetilde{g}^{\prime}}^{2}\right)=\widehat{\widetilde{g}}^{\prime}\left(x^{*}\right)$. Therefore $\widetilde{g}^{\prime}(d \tilde{x})=\rho(\tilde{x}) g(d \tilde{x})$ and $\widetilde{g}^{\prime} \cong \widetilde{g}$.

Lastly we shall give an example which is not equivalent to any representation with a real 1-cocycle.

Example 5. Under the same notation in this section, let $g$ be a Gaussian 
measure on $(X, \mathfrak{B}), H_{g}$ be the reproducing kernel space, and $h_{1}, \cdots, h_{n}, \cdots$ be a c.o.n.s. in $H_{g}$. Then for any $\varphi \in H_{g}$

$$
\sum_{n=1}^{\infty}\left\{W_{h_{n}}(x-\varphi)^{3}-3 W_{h_{n}}(x-\varphi)-W_{h_{n}}^{3}(x)+3 W_{h_{n}}(x)\right\}
$$

converges for $g$-a.e. $x$, because it is equal to

$$
\sum_{n=1}^{\infty}\left\{-3\left(W_{h_{n}}^{2}(x)-1\right)\left\langle\varphi, h_{n}\right\rangle_{H}+3 W_{h_{n}}(x)\left\langle\varphi, h_{n}\right\rangle_{H}^{2}-\left\langle\varphi, h_{n}\right\rangle_{H}^{3}\right\}
$$

and it is well known that $\sum_{n=1}^{\infty} a_{n}\left(X_{n}^{2}(\omega)-1\right), \sum_{n=1}^{\infty} a_{n} X_{n}(\omega)$ converges if and only if $\left\{a_{n}\right\}_{n} \in l^{2}$, respectively, where $\left\{X_{n}(\omega)\right\}_{n}$ is i.i.d. random variables which obey to the normal law $N(0,1)$. Put for $c \in \boldsymbol{R}$,

$$
\zeta_{c}(x, \varphi):=\exp \left(i c \sum_{n=1}^{\infty}\left(W_{h_{n}}^{3}(x-\varphi)-3 W_{h_{n}}(x-\varphi)-W_{h_{n}}^{3}(x)+3 W_{h_{n}}(x)\right) .\right.
$$

Then $\zeta_{c}$ is a 1-cocycle. Now consider a representation of $H_{g}$ such that

$$
V_{g, c}(\varphi): h(x) \in \mathrm{L}_{g}^{2} \longmapsto \sqrt{\frac{d g_{\varphi}}{d g}}(x) \zeta_{c}(x, \varphi) h(x-\varphi) \in \mathrm{L}_{g}^{2}
$$

Let us calculate the value $\left\langle V_{g, c}(\varphi) h, g\right\rangle_{2}$.

$$
\begin{aligned}
& \left\langle V_{g, c}(\varphi) h, k\right\rangle_{2}=\int_{X} \exp \left(-4^{-1}\|\varphi\|_{H_{g}}^{2}+2^{-1} W_{\varphi}(x)\right) \cdot \\
& \quad \exp \left(i c \sum_{n=1}^{\infty}\left(W_{h_{n}}^{3}(x-\varphi)-3 W_{h_{n}}(x-\varphi)-W_{h_{n}}^{3}(x)+3 W_{h_{n}}(x)\right)\right) h(x-\varphi) \bar{g}(x) g(d x) \\
& =\prod_{n=N+1}^{\infty} \exp \left(-4^{-1} \varphi_{n}^{2}\right) \int_{-\infty}^{\infty} \exp \left(2^{-1} \varphi_{n} t\right) \exp \left(i c\left(-3 t^{2} \varphi_{n}+3 t \varphi_{n}^{2}-\varphi_{n}^{3}+3 \varphi_{n}\right)\right) \cdot \\
& (2 \pi)^{-1 / 2} \exp \left(-2^{-1} t^{2}\right) d t \cdot \int_{R^{N}} \int_{R^{m}} \exp \left(i \sum_{n=1}^{N} \varphi_{n} x_{n}\right) \mathcal{F}\left\{( 2 \pi ) ^ { - N / 4 } \operatorname { e x p } \left(\sum _ { n = 1 } ^ { N } \left(-4^{-1} \xi_{n}^{2}\right.\right.\right. \\
& \left.\left.+i c\left(\xi_{n}^{3}-3 \xi_{n}\right)\right)\right) \cdot \\
& \left.H\left(\xi_{1}, \cdots, \xi_{N}, y_{1}, \cdots, y_{M}\right)\right\}(x) \bar{F}\left\{(2 \pi)^{-N / 4} \exp \left(\sum_{n=1}^{N}\left(4^{-1} \xi_{n}^{2}+i c\left(\xi_{n}^{3}-3 \xi_{n}\right)\right)\right) \cdot\right. \\
& \left.G\left(\xi_{1}, \cdots, \xi_{N}, y_{1}, \cdots, y_{M}\right)\right\}(x) d x g_{M}(d y),
\end{aligned}
$$

where $\varphi_{n}=\left\langle\varphi, h_{n}\right\rangle_{H g}$. Hereafter we shall proceed a similar manner as before and obtain a spectral measure $\sigma_{c}$ on $\left(H_{g}^{a}, \mathfrak{\mho}_{H g}\right)$ whose characteristic function is

$$
\widehat{\sigma}_{c}(\varphi)=\prod_{n=1}^{\infty} \int_{-\infty}^{\infty} \exp \left(i \varphi_{n} t\right)(2 \pi)^{-1 / 2}\left|\mathcal{F}\left\{\exp \left(-4^{-1} \xi^{2}+i c\left(\xi^{3}-3 \xi\right)\right)\right\}\right|^{2}(t) d t
$$

(The spectral measure is attained at $h=1$.)

By the way, if $\left(V_{g, c}, H_{g}\right)$ would be equivalent to some $\left(V_{\mu, \theta}, H_{g}\right)$ with a real 1-cocycle $\theta$, then $\sigma_{c}$ must be quasi-invariant under the map $-\mathrm{I}: \varphi^{a} \longmapsto-\varphi^{a}$. Because we have $\left\langle V_{\mu, \theta}(\varphi) h, h\right\rangle_{2}=\left\langle V_{\mu, \theta}(-\varphi) h, h\right\rangle_{2}$ for all real valued functions $h \in \mathrm{L}_{\mu}^{2}$ from which the invariance of the spectral measure $\sigma_{\mu, \theta}$ follows. Since $\sigma_{c}$ is regarded as a product measure of the countable copies of a 1-dimensional 
measure, so by the theorem of Kakutani (See, [5]),

$$
\rho_{c}(t):=\left|\mathcal{F}\left\{\exp \left(-4^{-1} \xi^{2}\right) \exp \left(i c\left(\xi^{3}-3 \xi\right)\right)\right\}\right|^{2}(t)
$$

must be an even function of $t$. Consequently,

$$
\psi_{c}(u):=\int_{-\infty}^{\infty} \exp (i t u) \rho_{c}(t) d t
$$

is a real valued function. We shall calculate it exactly.

$$
\begin{aligned}
\psi_{c}(u)= & (2 \pi)^{-1 / 2} \int_{-\infty}^{\infty} \exp \left(-2^{-1}\left(t-2^{-1} u\right)^{2}-8^{-1} u^{2} .\right. \\
& \left.+i c\left[(t-u)^{3}-3(t-u)-t^{3}+3 t\right]\right) d t \\
= & (2 \pi)^{-1 / 2} \exp \left(-8^{-1} u^{2}+i c\left[3 u-4^{-1} u^{3}\right]\right) \int_{-\infty}^{\infty} \exp \left(-2^{-1} t^{2}-3 i c u t^{2}\right) d t \\
= & \exp \left(-8^{-1} u^{2}+i c\left[3 u-4^{-1} u^{3}\right]-2^{-1} \log (1+6 c i u)\right) \\
= & \exp \left(-8^{-1} u^{2}-4^{-1} \log \left(1+36 c^{2} u^{2}\right)+i\left[c\left(3 u-4^{-1} u^{3}\right)-2^{-1} \tan ^{-1} 6 c u\right]\right) .
\end{aligned}
$$

Thus $\psi_{c}(u)$ has a non-zero imaginary part for each $c$, which is a contradiction. Since $\psi_{c}$ is different for each $c$, so $\sigma_{c}$ is singular with each other, and $\left(V_{g, c}, H\right)$ is a different representation for each $c$.

\section{References}

[1] Diximier., J. Von Neumann algebras, North-Holland Mathematical Library, 27 (1981).

[2] Feldeman, J., Equivalence and perpendicularity of Gaussian processes, Pacific J. Math., 8 (1958), 128-137.

[3] Gel'fand, J.M. and Vilenkin, N.Y., Generalized functions 4, Academic press (1964).

[4] Ito, K., Probabilty theory, Iwanami (1953) (in Japanese).

[5] Kakutani, S., On equivalence of infinite product measures, Ann. of Math., 49 (1948), 214224.

[6] Neumann, V.J., On infinite direct products, Composition Math., 6 (1938), 1-77.

[7] Okazaki, Y. and Takahashi, Y., The ergodicity of the convolution $\mu * \nu$ on a vector space, Publ. RIMS. Kyoto Univ., 23 (1987), 727-730.

[8] Sakai, S., $C^{*}$-algebras and $W^{*}$-algebras, Ergebnisse der mathematic und ihrer grenzgebiete, 60 (1971).

[9] Shimomura, H., Some results on quasi-invariant measures on infinite dimensional space, $J$. of Math. Kyoto Univ., 24 (1981), 703-713.

[10] Talagrand, M., Mesures Gaussiennes sur un espace localement convexe, Z. Wahrsheinlichkeitstheorie Verw. Gebiete, 64 (1983), 181-209.

[11] Yamasaki, Y., Measures on infinite dimensional spaces 2, Kinokuniya (1978) (in Japanese).

Added in proof: After writing this paper, the author was informed that there is a counterexample, which is stated below, for the ergodicity of Gaussian measures on linear spaces which is due to Bogachev. 
Example: By a result of Fremlin (See the following reference A.1), there exist a centered Gaussian measure $g$ on a Banach space $X$ and $x_{0}^{*} \in X^{*}$ such that $R_{\gamma} x_{0}^{*}$ is not in $X$, where $R_{r} x_{0}^{*}$ is defined as a functional on $X^{*}$ by

$$
R_{r} x_{0}^{*}\left(x^{*}\right)=\int_{X}\left\langle x, x^{*}\right\rangle\left\langle x, x_{0}^{*}\right\rangle g(d x) .
$$

Since $X$ is a Banach space, the set $E:=\left\{w \in W \mid R_{\gamma} w \in H\right\}$ is a closed linear space of the Hilbert space $W$. Let $x_{E}^{*}$ be the orthogonal projection of $x_{0}^{*}$ to $E$ and put $w_{0}:=x_{0}^{*}-x_{E}^{*}$. Then for any $h \in H, w_{0}(x+h)=w_{0}(x)$ holds for $g$-a.e. $x$, which is easily verified. Hence there exists a non-trivial $H$-invariant function, so $g$ is not $H$-ergodic.

Thus the first question of Remark often Proposition 6.1 is solved negatively.

A.1 Fremlin, D.H. and Talagrand, M., A Gaussian measure on $l^{\infty}$, Ann. Probab., 8 (1980), 1192-1193.

A.2 Bogachev., V.I., Gaussian measures on linear spaces, preprint. 
